\title{
Screening and identification of biomarkers for systemic sclerosis via microarray technology
}

\author{
CHEN XU $^{1 *}$, LING-BING MENG $^{2 *}$, YU-CHEN DUAN $^{1}$, YONG-JING CHENG ${ }^{1}$, \\ CHUN-MEI ZHANG ${ }^{1}$, XING ZHOU $^{1}$ and CI-BO HUANG ${ }^{1}$ \\ Departments of ${ }^{1}$ Rheumatology and ${ }^{2}$ Neurology, \\ Beijing Hospital, National Center of Gerontology, Beijing 100730, P.R. China
}

Received June 5, 2019; Accepted August 13, 2019

DOI: $10.3892 /$ ijmm.2019.4332

\begin{abstract}
Systemic sclerosis (SSc) is a complex autoimmune disease. The pathogenesis of SSc is currently unclear, although like other rheumatic diseases its pathogenesis is complicated. However, the ongoing development of bioinformatics technology has enabled new approaches to research this disease using microarray technology to screen and identify differentially expressed genes (DEGs) in the skin of patients with SSc compared with individuals with healthy skin. Publicly available data were downloaded from the Gene Expression Omnibus (GEO) database and intra-group data repeatability tests were conducted using Pearson's correlation test and principal component analysis. DEGs were identified using an online tool, GEO2R. Functional annotation of DEGs was performed using Gene Ontology (GO) and the Kyoto Encyclopedia of Genes and Genomes (KEGG) analysis. Finally, the construction and analysis of the protein-protein interaction (PPI) network and identification and analysis of hub genes was carried out. A total of 106 DEGs were detected by the screening of SSc and healthy skin samples. A total of 10 genes [interleukin-6, bone morphogenetic protein 4, calumenin (CALU), clusterin, cysteine rich angiogenic inducer 61 , serine protease 23 , secretogranin II, suppressor of cytokine signaling 3, Toll-like receptor 4 (TLR4), tenascin C] were identified as hub genes with degrees $\geq 10$, and which could sensitively and specifically predict SSc based on receiver operator characteristic curve analysis. GO and KEGG analysis showed that variations in hub genes were mainly enriched in positive regulation of nitric oxide biosynthetic processes, negative regulation
\end{abstract}

Correspondence to: Dr Ci-Bo Huang, Department of Rheumatology, Beijing Hospital, National Center of Gerontology, 1 Dahua Road, Dong Dan, Beijing 100730, P.R. China

E-mail: huangcibo1208@139.com

${ }^{*}$ Contributed equally

Key words: systemic sclerosis, biomarkers, hub genes, microarray technology, differentially expressed genes of apoptotic processes, extracellular regions, extracellular spaces, cytokine activity, chemo-attractant activity, and the phosphoinositide 3 kinase-protein kinase B signaling pathway. In summary, bioinformatics techniques proved useful for the screening and identification of biomarkers of disease. A total of 106 DEGs and 10 hub genes were linked to SSc, in particular the TLR4 and CALU genes.

\section{Introduction}

Systemic sclerosis ( $\mathrm{SSc}$ ), also called systemic scleroderma, is a complex autoimmune disease. Pathological features of SSc include skin and visceral fibrosis, vascular alterations and auto-antibodies against various cellular antigens (1). The available data show the prevalence of SSc to be 50-300 cases/per million population/per year, with the incidence of new cases being 2.3-22.8 cases/per million population/per year (2). Despite the rarity of the disease, the mortality rate of SSc is higher compared with other rheumatic diseases, especially diffuse cutaneous systemic sclerosis (dcSSc) $(3,4)$.

The pathogenesis of SSc is currently unclear, although as with other rheumatic diseases its pathogenesis is complicated. SSc may be caused by genetic susceptibility or by environmental factors (1). Genetic association studies and sequencing analysis have identified factors that may lead to genetic susceptibility to SSc and its specific complications $(5,6)$. Gene expression profiling of the whole transcriptome is increasingly being used to explore disease-related genes and enable disease classification and clinical prediction $(5,6)$. Derrett-Smith et al $(7)$ conducted gene expression profiling analysis of unaffected skin obtained from patients with localized cutaneous systemic sclerosis (lcSSc). The results showed that the differentially expressed genes (DEGs) are related to cardiovascular system and mainly enriched in fibrotic signaling pathways. It may be helpful to explain the mechanisms of vascular complications in SSc. Gardner et al (8) found that biopsy samples from patients with SSc had a robust and unique gene expression profile. A total of $\sim 1,800$ candidate genes can be used to distinguish between lesioned skin and normal skin $(\mathrm{P}<0.05)$. Therefore, gene expression analysis of SSc provides a possible means to gain insights into its pathogenesis. Furthermore, it can also provide clues and ideas for exploring potential therapeutic targets. 
High-throughput sequencing and microarray technology offer ideal tools for profiling large gene expression datasets in order to gain a comprehensive understanding of the mechanisms underlying various diseases. For example, by microarray technology, the expression levels of microRNAs (miRNAs) can be evaluated in the tissue samples from patients and in the normal tissue. This analysis can reveal a group of differentially expressed miRNAs. With further functional studies and downstream targeted genes and pathways recognition, specific miRNAs could be identified as candidate biomarkers related to disease pathogenesis or progression (9). The same can be done by computational approaches (10-12). Through the integrated analysis of publicly available bioinformatics datasets can also reach the starting point of identifying effective markers for disease diagnosis and prognosis (13).

In recent years, with the continuous development of bioinformatics technology, a series of different analytical methods have been used for researching disease processes, based on differentially expressed genes (DEGs). For the present study two datasets, GSE95065 and GSE76885, were downloaded from the Gene Expression Omnibus (GEO) database, the sample sources of which were from SSc patients with affected skin tissue and healthy control (HC) skin tissue. GEO2R (http://www.ncbi.nlm.nih.gov/geo/geo2r/) is an interactive online tool that can be used to identify DEGs by comparing samples from GEO series (14). GEO2R was used to screen DEGs. Then, the biological processes (BP), cell components (CC), molecular functions (MF) and signal pathways the two groups of DEGs are involved in were investigated by Gene Ontology (GO) and Kyoto Encyclopedia of Genes and Genomes (KEGG) analysis. Next, by framing the protein-protein interaction (PPI) network and filtering the significant modules of this network it was possible to define the top-ten hub genes. The aims of the present study were to identify novel biomarkers and potential therapeutic targets for SSc.

\section{Materials and methods}

Access to public data. The GEO database (http://www.ncbi. nlm.nih.gov/geo) is an open functional genomics database of high-throughput resources, including microarrays, gene expression data and ChIP-seq data (15). The selection criteria for this study were GEO datasets compilated of skin biopsy expression data obtained genome-wide from patients with SSc and the exclusion criteria was samples from patients with lcSSc. The Search details were 'Scleroderma, Systemic'[Mesh] AND 'Homo sapiens'[porgn] AND ('gse'[Filter] AND 'Expression profiling by array'[Filter] AND 'attribute name tissue'[Filter]) and revealed 66 different results relative to SSc gene expression datasets. After having other tissues or diseases filtering out, two expression profiling datasets, GSE95065 [GPL23080 (HG-U133A_2) Affymetrix Human Genome U133A 2.0 Array] (16) and GSE76885 (GPL6480 Agilent-014850 Whole Human Genome Microarray 4x44K G4112F) (https://www. ncbi.nlm.nih.gov/geo/query/acc.cgi?acc=GSE76885), were downloaded from the GEO database. The probes were transformed into homologous gene symbols by means of the platform's annotation information. The GSE95065 dataset was based on 15 control (Con) skin tissue samples from healthy individuals and 18 skin tissue samples from patients with SSc.
The GSE76885 dataset was based on 18 Con skin tissue samples from healthy individuals and 59 skin tissue samples from patients with SSc. The samples were taken from an in silico approach. Although GSE76885 was not specific to dcSSc or lcSSc, there was a strong correlation among the samples in the SSc group according to the results of the present study, dcSSc is more common than $1 \mathrm{CSSc}$, as in clinical practice so this was taken into account. The overall design of GSE117928 was performed on peripheral blood mononuclear cells, GSE73674 was on endothelial progenitor cell-derived endothelial cells and GSE81292 on lung tissues. GSE76809 was a SuperSeries composed of SubSeries. Of these SubSeries, GSE9285 contained gene expression profiling of diffuse scleroderma and limited scleroderma; GSE32413, GSE45485, GSE59785, GSE68698 were not genome-wide datasets; samples of other series were from lcSSc or not from skin tissue.

Intra-group data repeatability test. The Pearson's correlation test was performed to verify intra-group data repeatability in the per group. The $\mathrm{R}$ programming language was used to provide the software and operating environment for statistical analysis and drawing of graphs. Correlations between all samples from the same dataset were visualized using heat maps which were also drawn using R. Principal component analysis (PCA) is a commonly used method for sample clustering and is often used for gene expression, diversity analysis, resequencing, and other sample clustering based on various variable information. The intra-group data repeatability of the dataset was tested by sample clustering analysis.

Identification of DEGs. GEO2R is an interactive web tool that allows users to compare two or more groups of samples in a GEO series in order to identify genes that are differentially expressed across experimental conditions. Results are presented as a table of genes ordered by significance. GEO2R performs comparisons on original submitter-supplied processed data tables using the GEOquery and limma R packages from the Bioconductor project. Bioconductor is an open source software project (http://www.bioconductor.org/) based on the $\mathrm{R}$ programming language that provides tools for the analysis of high-throughput genomic data. The GEO query $R$ package parses GEO data into $R$ data structures that can be used by other R packages. The limma (Linear Models for Microarray Analysis) R package, which could perform the paired-samples $\mathrm{T}$ test, has emerged as one of the most widely used statistical tests for identifying differentially expressed genes (17). It handles a wide range of experimental designs and data types and applies multiple-testing corrections on P-values to help correct for the occurrence of false positives. Therefore, GEO2R provides a simple interface that allows users to perform R statistical analysis without command line expertise.

GEO2R was used to search for mRNAs (DEGs) that were differentially expressed between control tissue samples and SSc tissue samples. The cut-off criterion was a $\mathrm{P}<0.05$, whereas the fold-change was $\geq 1.5$ or $\leq-1.5$. Venn diagrams were used to determine the intersection between the two datasets to obtain DEGs in common. Volcano maps were drawn using the volcano plotting tool (https://shengxin.ren). The DEGs were then screened by introducing the two datasets into 
the FunRich (functional enrichment analysis tool; http://www. funrich.org/). Venn diagrams were delineated using an online Venn tool (http://bioinformatics.psb.ugent.be/webtools/Venn/), which could then be used to visualize common DEGs shared between the two datasets.

Functional annotation for DEGs using GO and KEGG analysis. DAVID (https://david.ncifcrf.gov/home.jsp; version 6.8 ) is an online analysis tool suite that includes the function of Integrated Discovery and Annotation (18). DAVID is an online analysis tool, which could perform GO and KEGG analysis. Gene Ontology (GO) is a widely used initiative in bioinformatics and covers three key biological aspects, including BP, CC, and MF (19). The KEGG (https://www.kegg. $\mathrm{jp} /$ ) is one of the most commonly used biological information databases in the world. To perform GO and KEGG analysis of DEGs, the DAVID online tool was used. Results were considered statistically significant if $\mathrm{P}<0.05$.

Construction and analysis of the PPI network. The Search Tool for the Retrieval of Interacting Genes (STRING) online database (http://string-db.org) can be used to predict and trace the PPI network once common DEGs have been imported into it. The STRING database was used for the construction of the PPI network of the DEGs. The free visualization software tool, Cytoscape (version 2.8) (20), was used to visualize PPI networks. Next, the Cytoscape plug-in Molecular Complex Detection tool (MCODE; version 1.5.1) was used to identify the most important module on the network map. The criteria for the MCODE analysis were that the degree of cut-off $=2$, MCODE scores $>5$, maximum depth $=100$, node score cut-off $=0.2$ and $\mathrm{k}$-score $=2(21)$.

Identification and analysis of hub genes. Once the degrees were set (degrees $\geq 10$ ), the hub genes were identified. Subsequently, following the GO and KEGG analysis using the DAVID database, functional annotation of the hub genes was performed. Correlation analysis between the hub genes was also carried out. A total of three hierarchical clustering heat-maps of hub gene expression were visualized using $\mathrm{R}$. Finally, receiver operator characteristic (ROC) curve analysis was performed to determine the usefulness of these hub genes for predicting SSc.

Statistical analyses. All statistical analyses were conducted using SPSS software (version 21.0; IBM Corps.). The Pearson's correlation coefficient was used to validate the intra-group data repeatability and to analyze correlation among hub genes. The two-sample t-test was used for comparing the mean values of two groups (SSc and control groups). ROC curve analysis was performed to determine the ability of the hub genes to predict SSc. $\mathrm{P}<0.05$ was considered to indicate a statistically significant difference.

\section{Results}

Validation of the datasets. To further validate the intra-group data repeatability, the Pearson's correlation test and PCA were employed. Based on the Pearson's correlation test, it was found that in the GSE95065 dataset there were strong correlations among the samples in the control group and that there were also strong correlations among the samples in the SSc group (Fig. 1A). Based on the PCA the intra-group data repeatability for GSE95065 was acceptable. The distances between the samples in the control group were close and the distances between samples in the SSc group were also close in the dimension of PC1 (Fig. 1B). Based on Pearson's correlation test, it was found that for GSE76885 there was a strong correlation among the samples in the control group and a strong correlation among the samples in the SSc group (Fig. 2A). The PCA showed the intra-group data repeatability to be acceptable in the GSE76885 dataset. The distances between per samples in the control group were close and distances between per samples in the SSc group were also close in the dimension of PC1 (Fig. 2B).

Identification of DEGs in SSc and control samples. The volcano plot analysis was performed to present the DEGs between the Con skin tissue samples and SSc tissue samples. In the volcano plot, all nodes present the DEGs between the Con and SSc group. When the DEGs conformed to the cut-off criterion $(\mathrm{P}<0.05$, whereas the fold-change was $\geq 1.5$ or $\leq-1.5)$, the nodes were significant and would be marked as green or red. The green nodes present the downregulated DEGs and the red nodes present the upregulated DEGs in the SSc group, compared with the Con group. A total of 2,041 and 880 DEGs were obtained from the GSE95065 and GSE76885 datasets, respectively. Volcano plots of GSE95065 and GSE76885 are shown in Fig. 3A and B. A Venn diagram showed that 106 DEGs were common to both datasets (Fig. 3C).

Functional and pathway enrichment analysis of DEGs. GO analysis consists of three items: $\mathrm{BP}, \mathrm{CC}$ and MF. The results of the GO analysis in the present study showed that variations in DEGS linked with BP were mainly enriched in extracellular matrix organization, positive regulation of gene expression, inflammatory responses, positive regulation of IL-6 production, actin cytoskeleton reorganization, responses to viruses and monocyte differentiation (Fig. 4A). Variations in DEGs linked with $\mathrm{CC}$ were significantly enriched in extracellular spaces, extracellular regions, extracellular matrix, extracellular exosomes, the perinuclear region of the cytoplasm, intracellular spaces, Golgi cisternae membranes, Golgi apparatus, cell surfaces, cell-cell junctions, host cells and COPI-coated vesicles $(\mathrm{P}<0.05)$. Regarding $\mathrm{MF}$, DEGs were significantly enriched in receptor binding, protein binding, integrin binding, oxidoreductase activity, transcription regulatory-region DNA binding, GDP binding, actin filament binding and GTPase activity $(\mathrm{P}<0.05)$. Analysis of KEGG pathways indicated that the top canonical pathways associated with DEGs were salmonella infection, legionellosis, cytokine-cytokine receptor interaction, TNF signaling pathways and insulin resistance.

PPI and module network construction and hub gene selection. Construction of the PPI network and the identification of significant modules was performed, with 101 edges and 66 nodes found to be in the PPI network in Fig. 5A and 21 edges and 7 nodes found to be in the significant module in Fig. 5B. A total of ten genes [interleukin-6 (IL6), bone morphogenetic protein 4 (BMP4), calumenin (CALU), clusterin (CLU), cysteine rich 
A
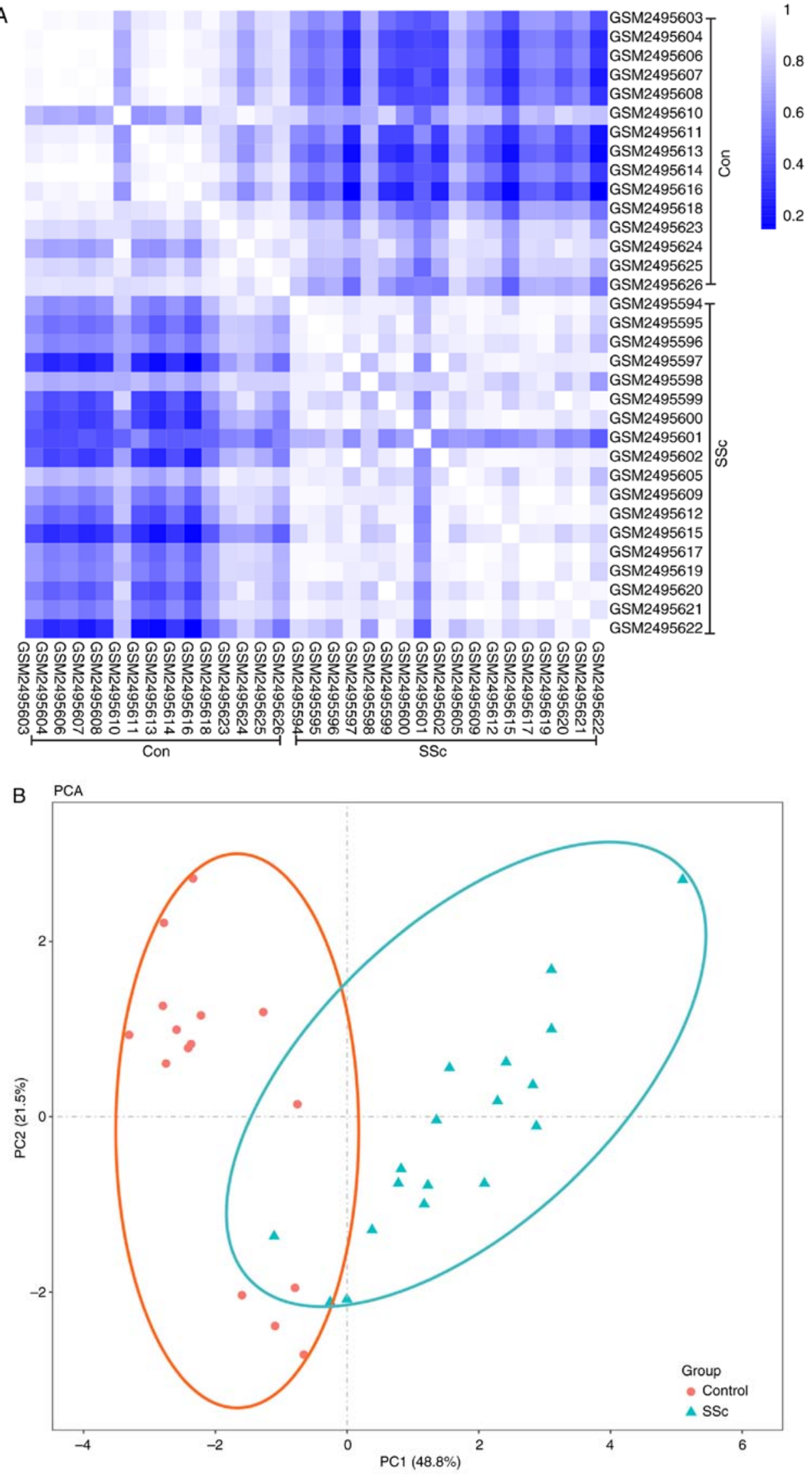

Figure 1. Intra-group data repeatability test for GSE95065 through the Pearson's correlation analysis and PCA. (A) Pearson's correlation analysis of samples from the GSE95065 dataset. The color reflects the intensity of the correlation. When $0<$ correlation $<1$, there exists a positive correlation. When $-1<$ correlation $<0$, there exists a negative correlation. The larger the absolute value of a number the stronger the correlation. (B) PCA of samples from the GSE95065 dataset. In the figure, $\mathrm{PC} 1$ and $\mathrm{PC} 2$ are used as the $\mathrm{X}$-axis and $\mathrm{Y}$-axis, respectively, to draw the scatter diagram, where each point represents a sample. In such a PCA diagram, the farther the two samples are from each other, the greater the difference is between the two samples in gene expression patterns. PC1, principal component 1; PC2, principal component 2; PCA, principal component analysis; SSc, Systemic sclerosis; Con, control. 
A
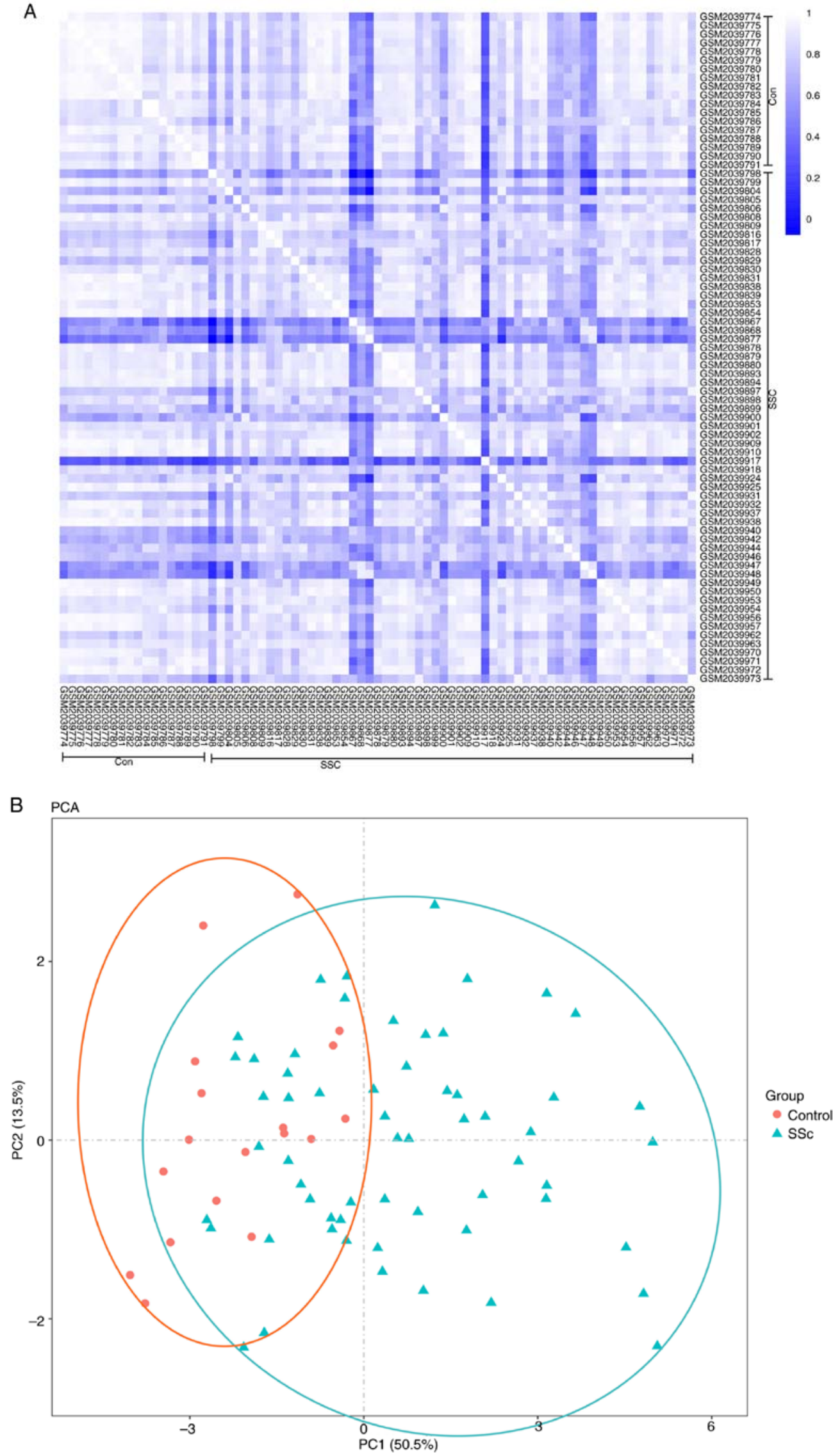

Figure 2. Intra-group data repeatability test for GSE76885 through the Pearson's correlation analysis and PCA. (A) Pearson's correlation analysis of samples from the GSE76885 dataset. The color reflects the intensity of the correlation. When $0<$ correlation $<1$, there exists a positive correlation. When $-1<$ correlation $<0$, there exists a negative correlation. The larger the absolute value of a number the stronger the correlation. (B) PCA of samples from the GSE76885 dataset. In the figure, $\mathrm{PC} 1$ and $\mathrm{PC} 2$ are used as the $\mathrm{X}$-axis and $\mathrm{Y}$-axis, respectively, to draw the scatter diagram, where each point represents a sample. In such a PCA diagram, the farther the two samples are from each other, the greater the difference is between the two samples in gene expression patterns. PC1, principal component 1; PC2, principal component 2; PCA, principal component analysis; SSc, systemic sclerosis. 
A

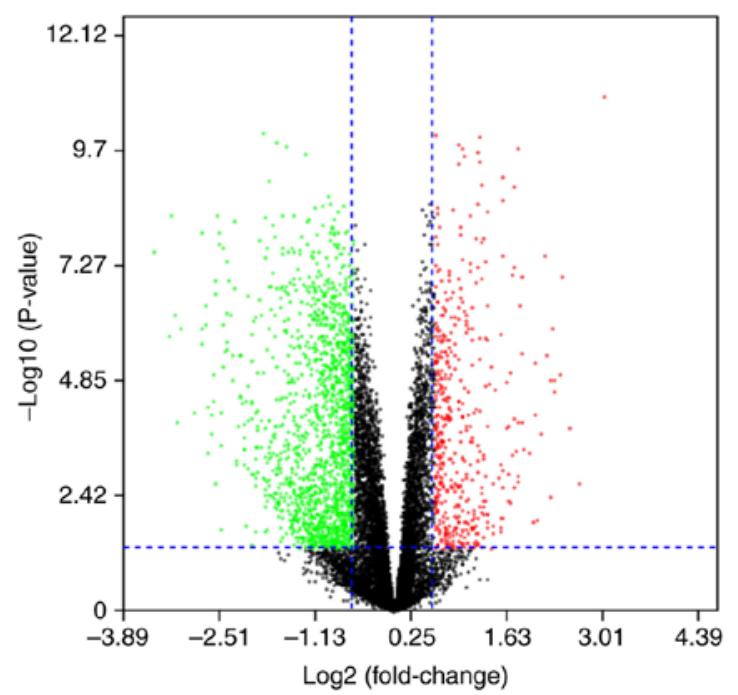

B

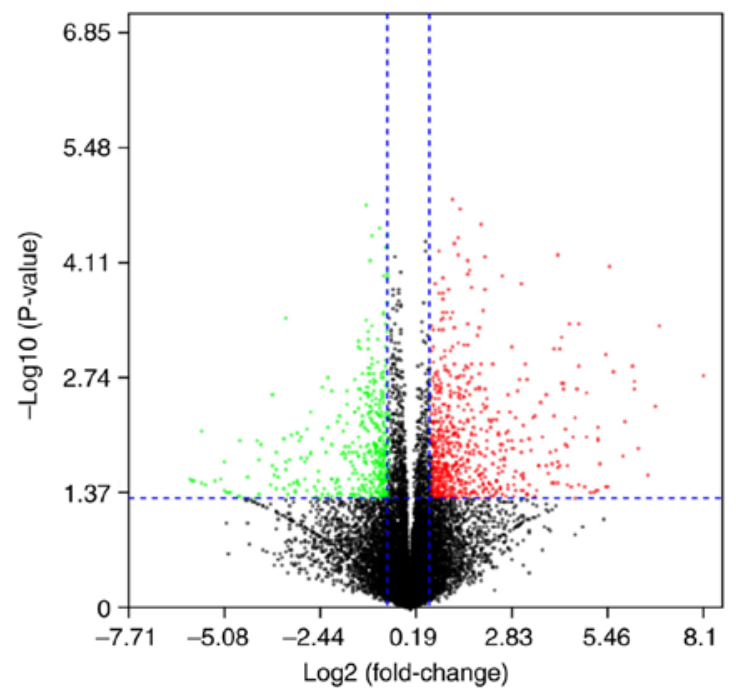

C

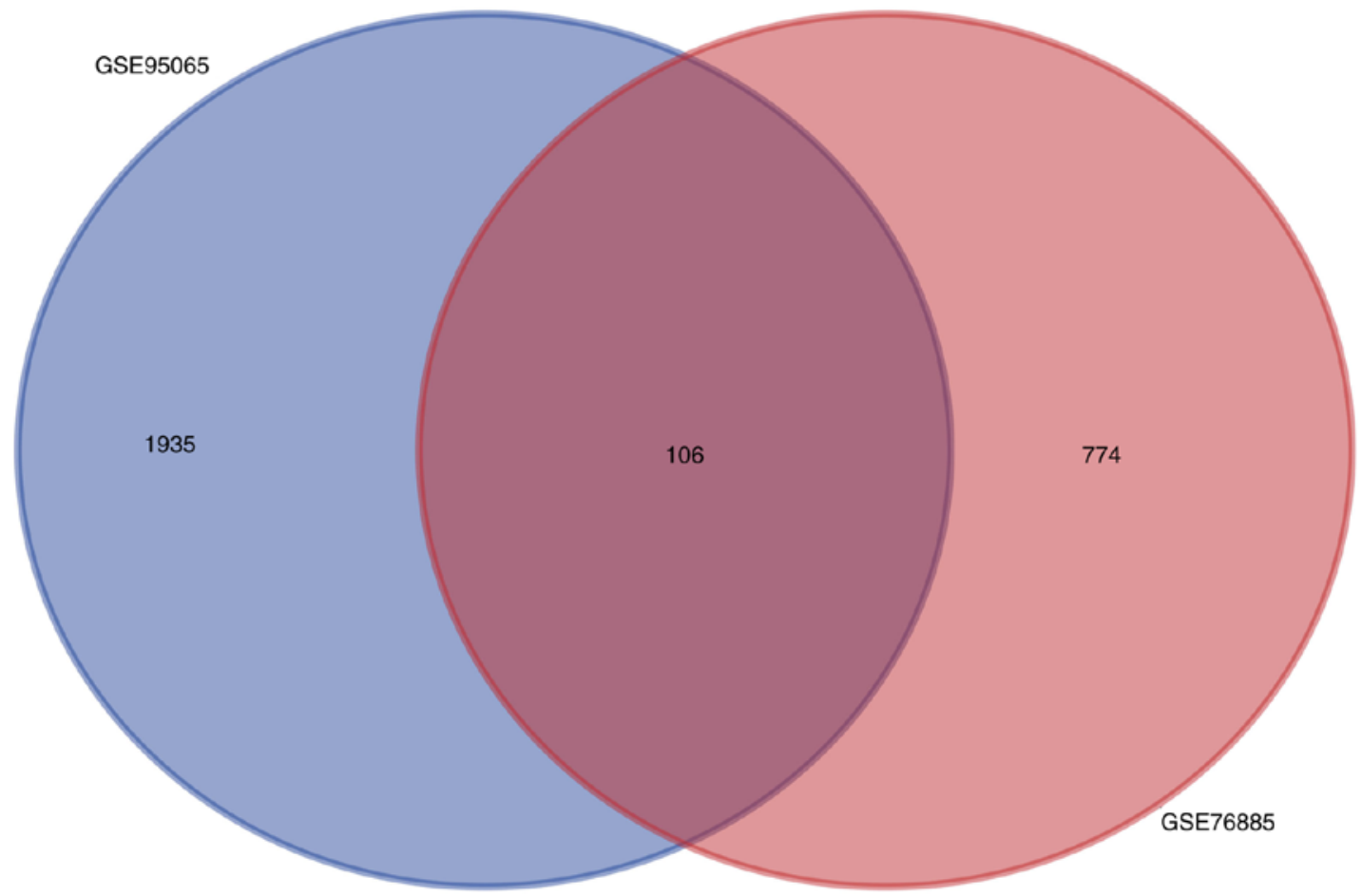

Figure 3. Identification of DEGs between skin samples from healthy control individuals and patients with SSc. (A) A volcano plot showing the difference between SSc and control skin samples after analysis of the GSE95065 dataset with GEO2R. The X-axis represents the fold-change (log-scaled) and the Y-axis represents the P-value (log-scaled). Each symbol represents a different gene. The red symbols represent upregulated genes; green symbols represent downregulated genes. (B) A volcano plot of the GSE76885 dataset. (C) A Venn diagram showing that 106 genes are shared by both the GSE95065 and the GSE76885 datasets. SSc, systemic sclerosis; DEG, differentially expressed genes.

angiogenic inducer 61 (CYR61), serine protease 23 (PRSS23), secretogranin II (SCG2), suppressor of cytokine signaling 3 (SOCS3), Toll-like receptor 4 (TLR4), tenascin C (TNC)] were identified as hub genes with degrees $\geq 10$ (Fig. 5C).

Hub gene analysis. The names, abbreviations and functions for the hub genes are shown in Table I.

These hub genes were analyzed using DAVID, GO and KEGG analysis. The results of these analyses showed that variations in BP of hub genes were mainly enriched in positive regulation of nitric oxide biosynthetic processes, positive regulation of osteoblast differentiation, negative regulation of apoptotic processes, osteoblast differentiation, positive regulation of NF- $\mathrm{KB}$ transcription factor activity, positive regulation of the extracellular signal regulated kinase (ERK)1 and ERK2 cascade, positive regulation of gene expression, positive regulation of cartilage development, and positive regulation of chemokine production (Fig. 6A). Variations in the $\mathrm{CC}$ of hub genes were significantly enriched in the extracellular region, extracellular space and extracellular matrix $(\mathrm{P}<0.01$; Fig. 6B). Variations in the MF of hub genes were significantly enriched in cytokine activity, and chemo-attractant activity, but were not significantly 
A

Top 20 of biological process enrichment

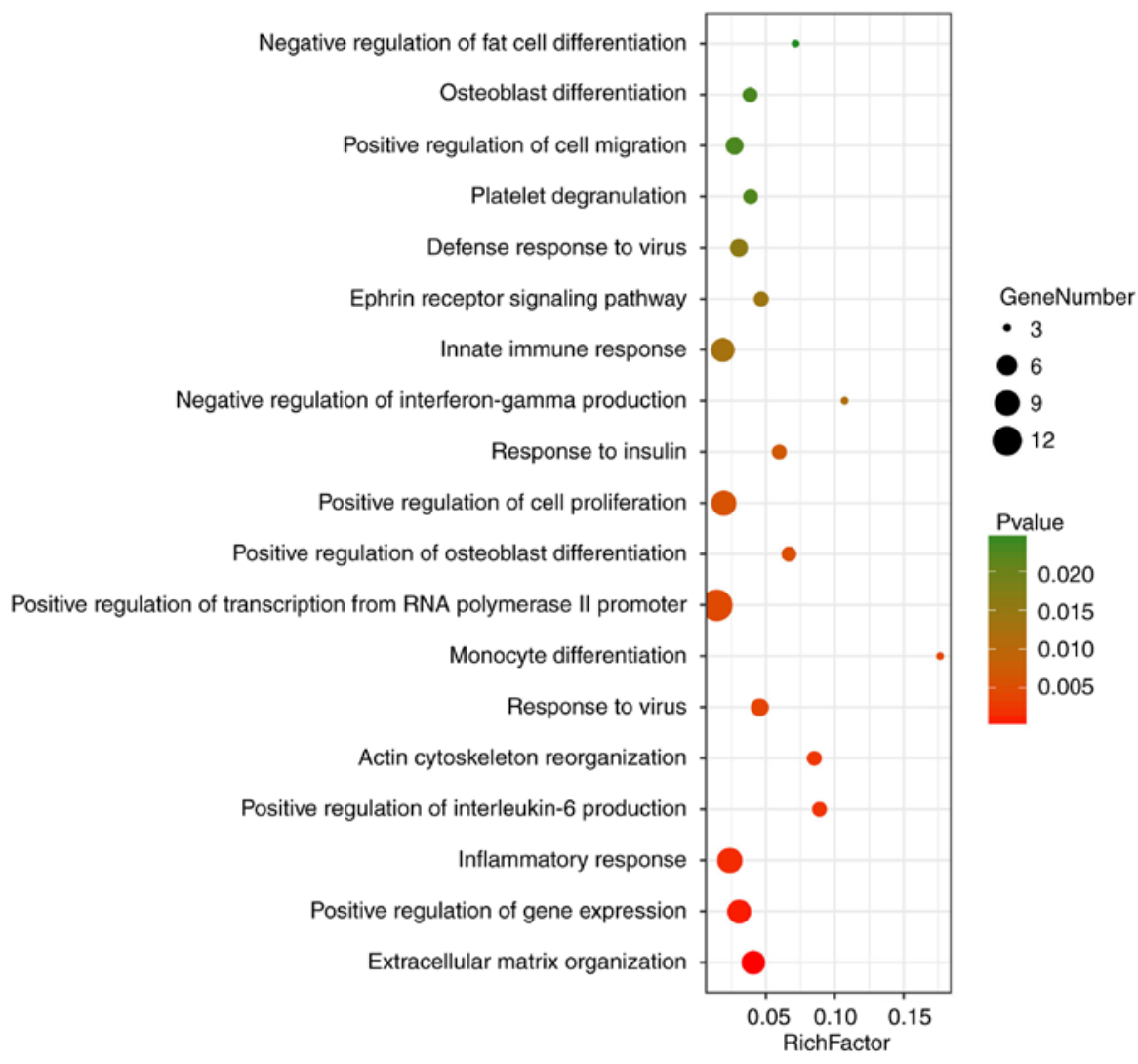

B

Top 13 of cellular component enrichment

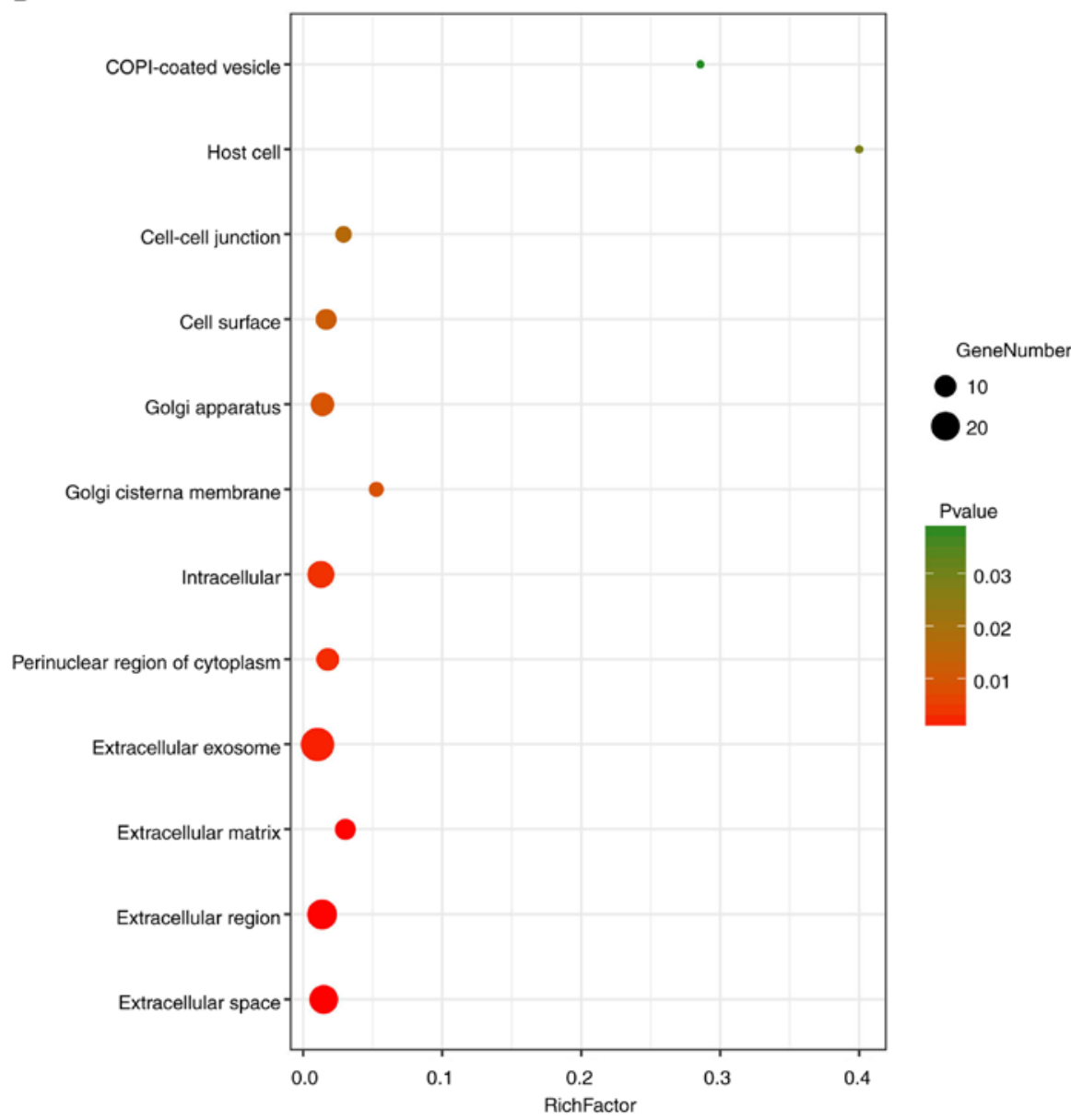

Figure 4. Enrichment analysis of the DEGs using the DAVID tool. Detailed information relating to changes in the (A) biological processes, (B) cellular components in SSc and control skin samples. 
C

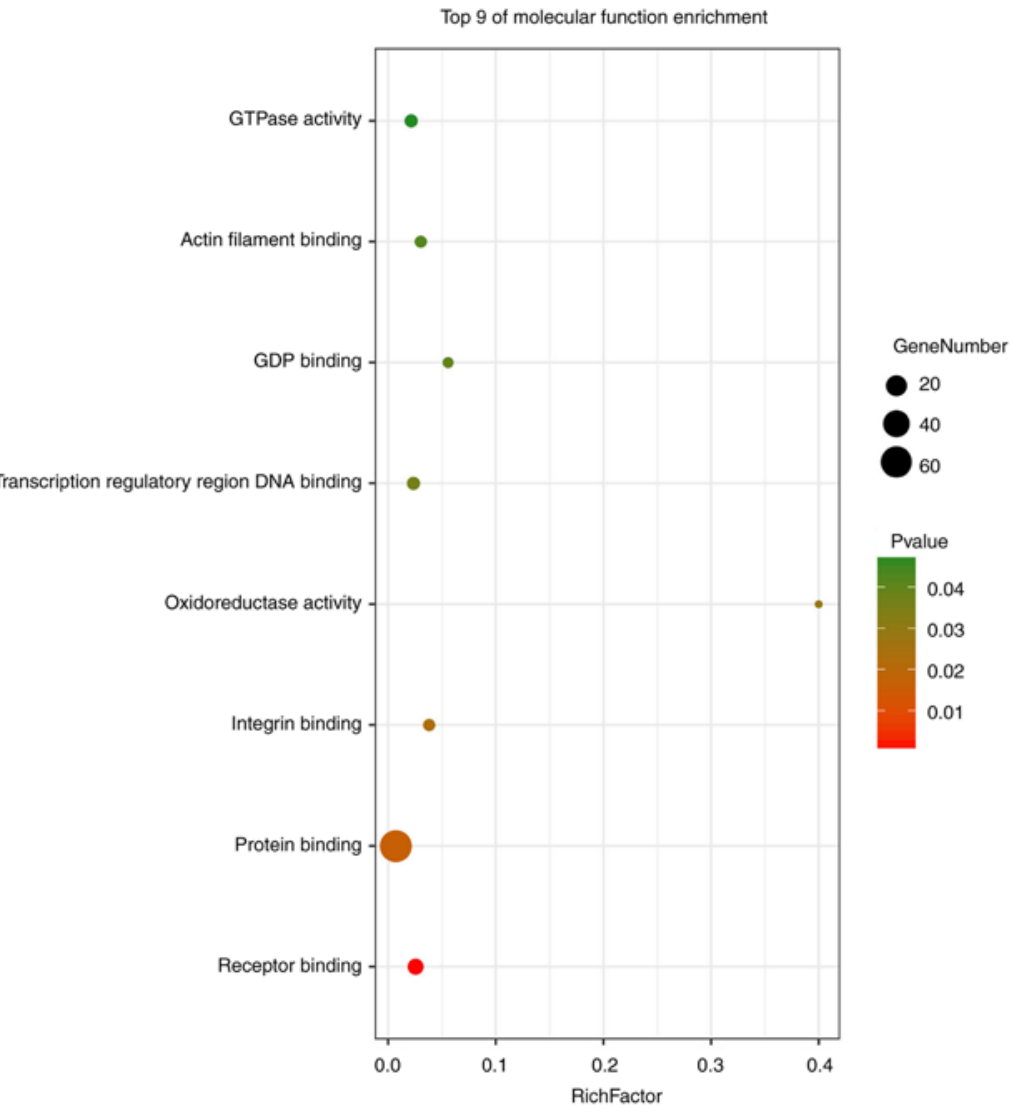

D

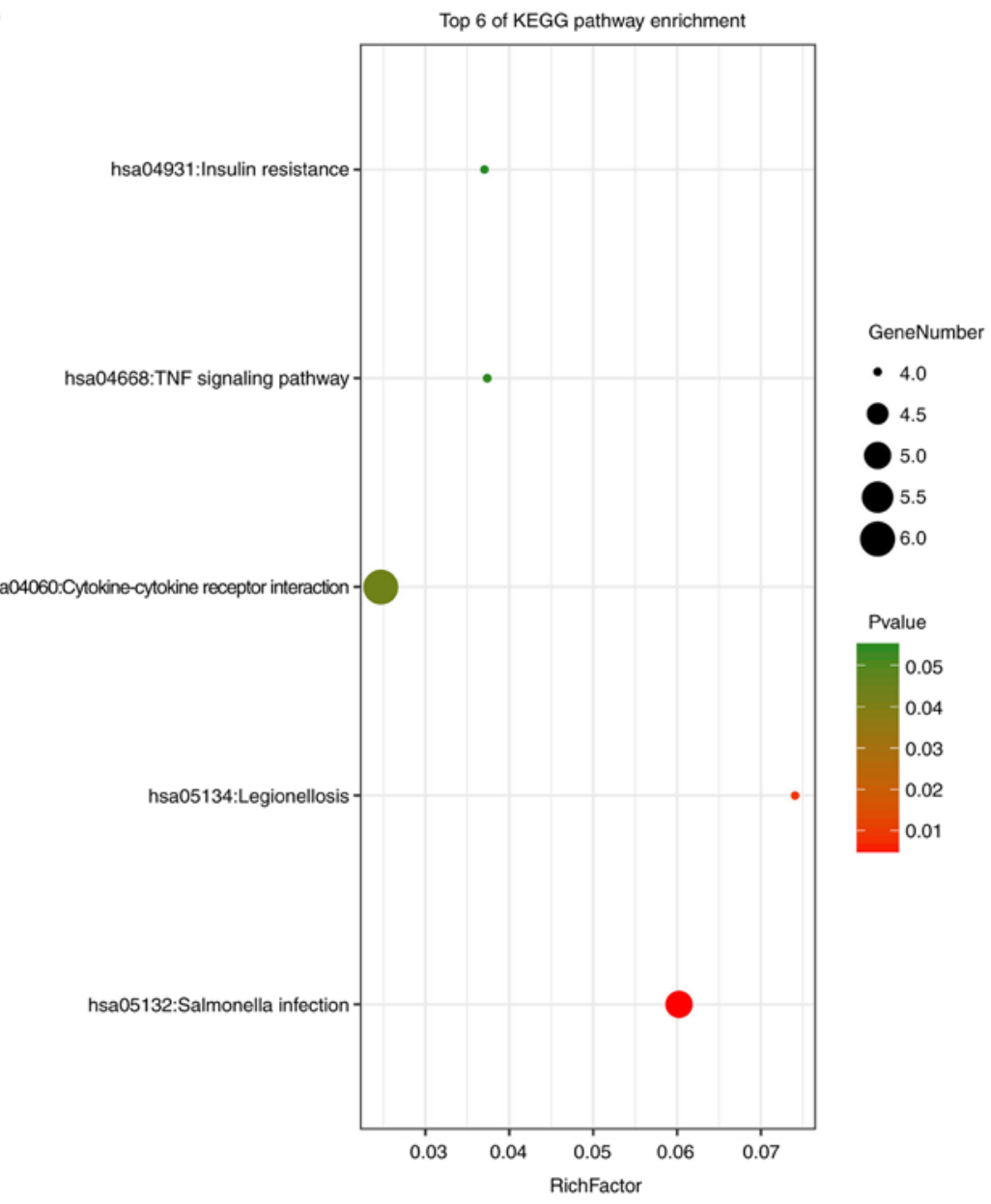

Figure 4. Continued. Enrichment analysis of the DEGs using the DAVID tool. Detailed information relating to changes in the (C) molecular functions of DEGs in SSc and control skin samples. (D) KEGG pathway analysis for DEGs. SSc, systemic sclerosis; DEGs, differentially expressed genes; KEGG, Kyoto Encyclopedia of Genes and Genomes. 

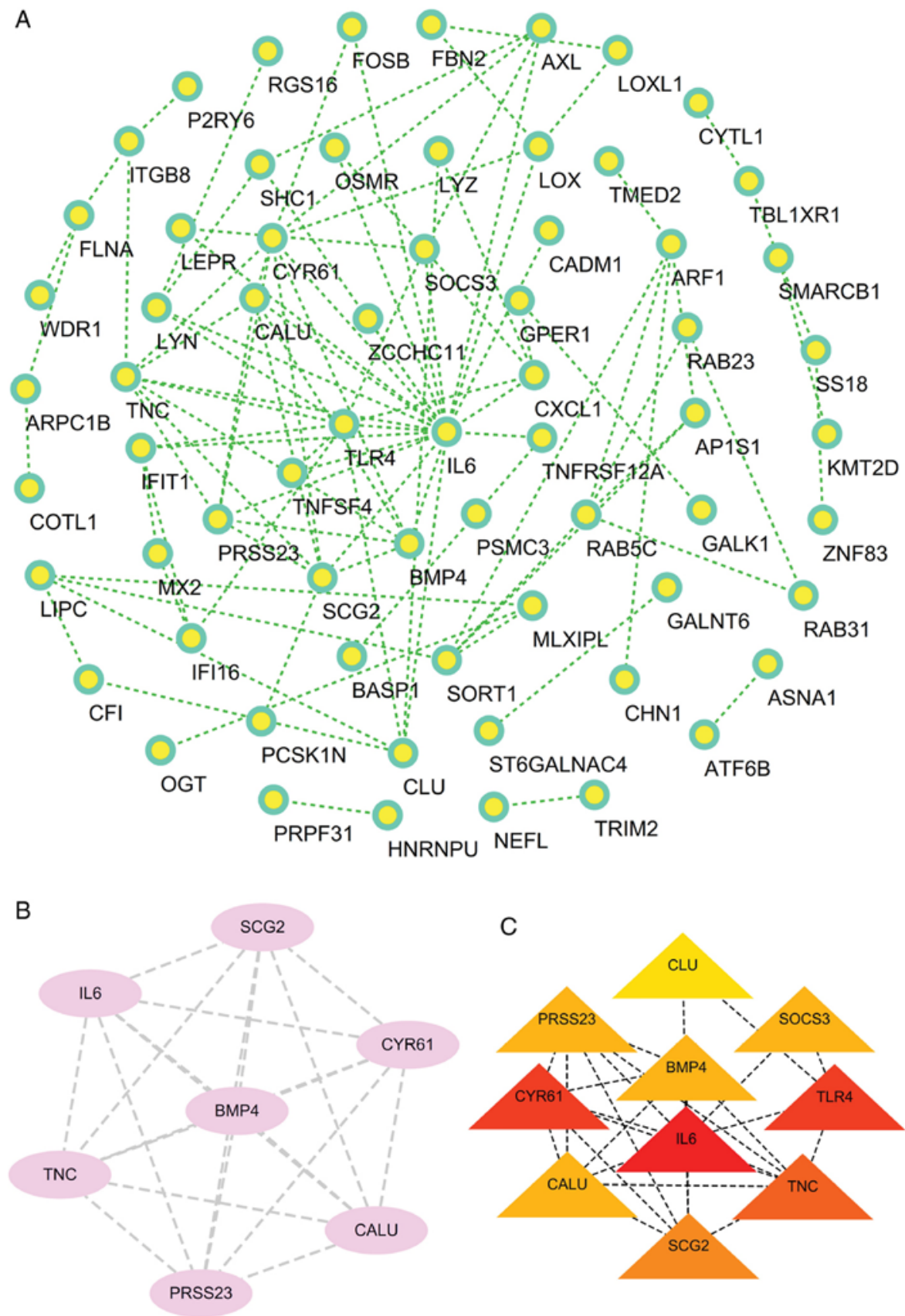

Figure 5. PPI network, significant module network, and the hub genes. (A) The PPI network showing the intricate relationships between DEGs. (B) The significant module network selected based on the PPI network. (C) A total of 10 genes (CLU, SOCS3, PRSS23, BMP4, TLR4, CYR61, IL6, CALU, TNC and SCG2) were identified as hub genes with degrees $\geq 10$. PPI, protein-protein interaction; DEGs, differentially expressed genes. IL6, interleukin-6; BMP4, bone morphogenetic protein 4; CALU, calumenin; CLU, clusterin; CYR61, cysteine rich angiogenic inducer 61; PRSS23, serine protease 23; SCG2, secretogranin II; SOCS3, suppressor of cytokine signaling 3; TLR4, Toll-like receptor 4; TNC, tenascin C.

enriched in heparin binding and growth factor activity (Fig. 6C). Analysis of KEGG pathways showed that hub genes were mainly enriched in influenza A infection, the PI3K-Akt signaling pathway, malaria, legionellosis, inflammatory bowel disease, pertussis infection, salmonella infection, rheumatoid arthritis, the HIF-1 signaling pathway and Chagas disease (Fig. 6D).

Heat maps showed that there was correlation between hub genes in the GSE95065 (Fig. 7A) and GSE76885 (Fig. 7B) datasets. Hierarchical clustering allowed for simple differentiation of SSc skin samples from the Con skin samples via the expression levels of hub genes in the GSE95065 (Fig. 8A) and GSE76885 (Fig. 8B) datasets.
ROC curve based on hub genes can be used to sensitively and specifically predict SSc. To identify accurate thresholds for hub genes to predict SSc ROC curves were constructed. The expression of all hub genes was associated with a diagnosis of SSc $(0.7<$ AUC $<1 ; \mathrm{P} \leq 0.05$; Table II; Fig. $8 \mathrm{C})$. The ROC curves of per hub genes are shown in Fig. 9.

\section{Discussion}

Pathological fibrosis is the most common and prominent feature of SSc. Since there is no treatment strategy for significantly delaying fibrosis, current treatment for SSc mainly focuses 
Table I. Summaries for the function of 10 hub genes.

\begin{tabular}{|c|c|c|c|c|}
\hline No. & $\begin{array}{c}\text { Gene } \\
\text { symbol }\end{array}$ & Full name & UniProtKB ID & Function \\
\hline 1 & IL6 & Interleukin 6 & P05231 (IL6_HUMAN) & $\begin{array}{l}\text { It is a potent inducer of the acute phase response. It } \\
\text { induces myeloma and plasmacytoma growth and induces } \\
\text { nerve cells differentiation. }\end{array}$ \\
\hline 2 & TLR4 & Toll-like receptor 4 & O00206 (TLR4_HUMAN) & $\begin{array}{l}\text { Toll-like receptors are single transmembrane cell-surface } \\
\text { receptors, which have a key role in the innate immune } \\
\text { system. }\end{array}$ \\
\hline 3 & CYR61 & $\begin{array}{l}\text { Cysteine rich angiogenic } \\
\text { inducer } 61\end{array}$ & O00622 (CCN1_HUMAN) & $\begin{array}{l}\text { Promotes cell proliferation, chemotaxis, angiogenesis } \\
\text { and cell adhesion. Appears to play a role in wound healing } \\
\text { by being upregulated in skin fibroblasts. }\end{array}$ \\
\hline 4 & $\mathrm{TNC}$ & Tenascin C & P24821 (TENA_HUMAN) & $\begin{array}{l}\text { Extracellular matrix protein implicated in guidance of } \\
\text { migrating neurons as well as axons during development, } \\
\text { synaptic plasticity as well as neuronal regeneration. } \\
\text { Promotes neurite outgrowth from cortical neurons grown } \\
\text { on a monolayer of astrocytes. }\end{array}$ \\
\hline 5 & SCG2 & Secretogranin II & P13521 (SCG2_HUMAN) & $\begin{array}{l}\text { Secretogranin- } 2 \text { is a neuroendocrine secretory granule } \\
\text { protein, which is the precursor for biologically active } \\
\text { peptides. }\end{array}$ \\
\hline 6 & SOCS3 & $\begin{array}{l}\text { Suppressor of cytokine } \\
\text { signaling } 3\end{array}$ & O14543 (SOCS3_HUMAN) & $\begin{array}{l}\text { Regulates IL- } 6 \text { signaling in vivo. Mediate the } \\
\text { ubiquitination and subsequent proteasomal degradation } \\
\text { of target proteins. }\end{array}$ \\
\hline 7 & BMP4 & $\begin{array}{l}\text { Bone morphogenetic } \\
\text { protein } 4\end{array}$ & P12644 (BMP4_HUMAN) & $\begin{array}{l}\text { Induces cartilage and bone formation. Acts in concert } \\
\text { with PTHLH/PTHRP to stimulate ductal outgrowth and } \\
\text { to inhibit hair follicle induction. }\end{array}$ \\
\hline 8 & CALU & Calumenin & O43852 (CALU_HUMAN) & $\begin{array}{l}\text { Involved in regulation of vitamin K-dependent } \\
\text { carboxylation of multiple } \mathrm{N} \text {-terminal glutamate residues. } \\
\text { Binds } 7 \text { calcium ions with a low affinity. }\end{array}$ \\
\hline 9 & PRSS23 & Serine protease 23 & O95084 (PRS23_HUMAN) & $\begin{array}{l}\text { This gene encodes a conserved member of the trypsin } \\
\text { family of serine proteases. Mouse experiments found a } \\
\text { decrease of mRNA levels of this gene after ovulation was } \\
\text { induced (49). }\end{array}$ \\
\hline 10 & CLU & Clusterin & P10909 (CLUS_HUMAN) & $\begin{array}{l}\text { Mitochondrial isoforms suppress BAX-dependent release } \\
\text { of cytochrome c into the cytoplasm and inhibit apoptosis. } \\
\text { Plays a role in the regulation of cell proliferation. }\end{array}$ \\
\hline
\end{tabular}

on mitigating symptoms and improving complications (22). Therefore, the exploration of the molecular mechanism that underlies SSc development and ways to identify patients at risk of severe organ involvement is of great importance to assist in the diagnosis, early treatment, and prognosis of SSc (23).

Gene expression profiling using microarray technology can provide information about the expression of thousands of genes in the human genome. For this study, several bioinformatics techniques were integrated to investigate data to screen and identify hub genes related to SSc. Two datasets, GSE95065 and GSE76885, were screened for DEGs and 106 DEGs were discovered that shared 10 hub genes in common: CLU, SOCS3, PRSS23, BMP4, TLR4, CYR61, IL6, CALU, TNC and SCG2. Among them, CLU, TLR4 and CALU were found to be differentially expressed and displayed better homogeneity between samples of SSc or samples of the Con group. Then a literature search was performed in Pubmed and it was found that the role of TLR4 in SSc was inconsistent with former studies and might be a 'bone of contention' (24-26). IL-6 has been a research hotspot in recent years. However, unlike TLR4 or IL-6, research into CALU has so far been limited. The results of the present study might represent a starting point for subsequent investigations into CALU. Therefore, the present study considered these three genes were significant and needed to be discussed.

TLR4 belongs to the family of pattern recognition receptors. As the first line of defense against infections, such receptors recognize pathogen-associated molecular patterns. TLR4 also binds to endogenous damage-associated molecular patterns (DAMPs) produced as a result of tissue damage. TLR4-mediated inflammation triggered by exogenous or endogenous ligands is involved in several diseases and plays a 
A

Top 21 of biological process enrichment

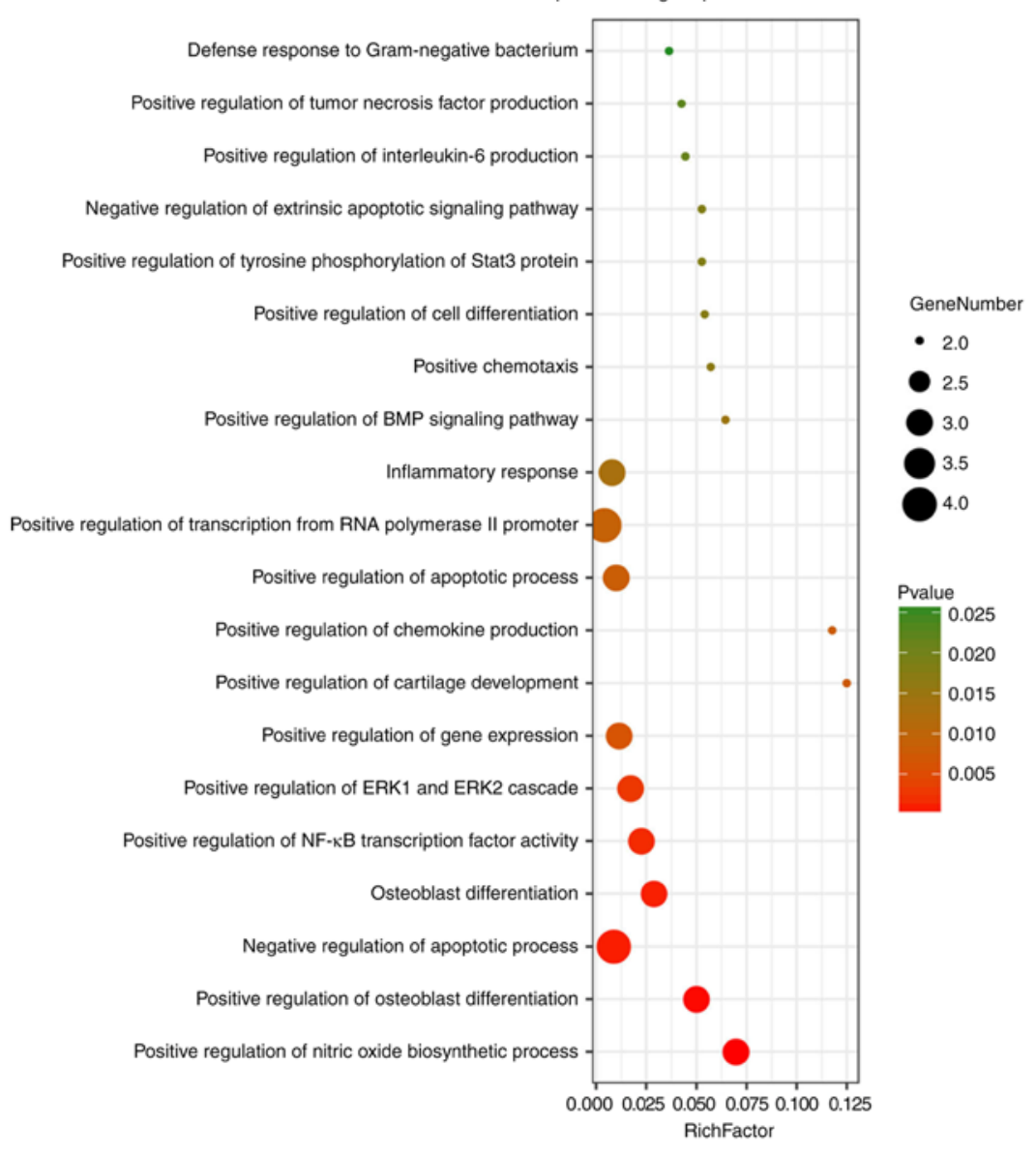

B

Top 4 of cellular component enrichment

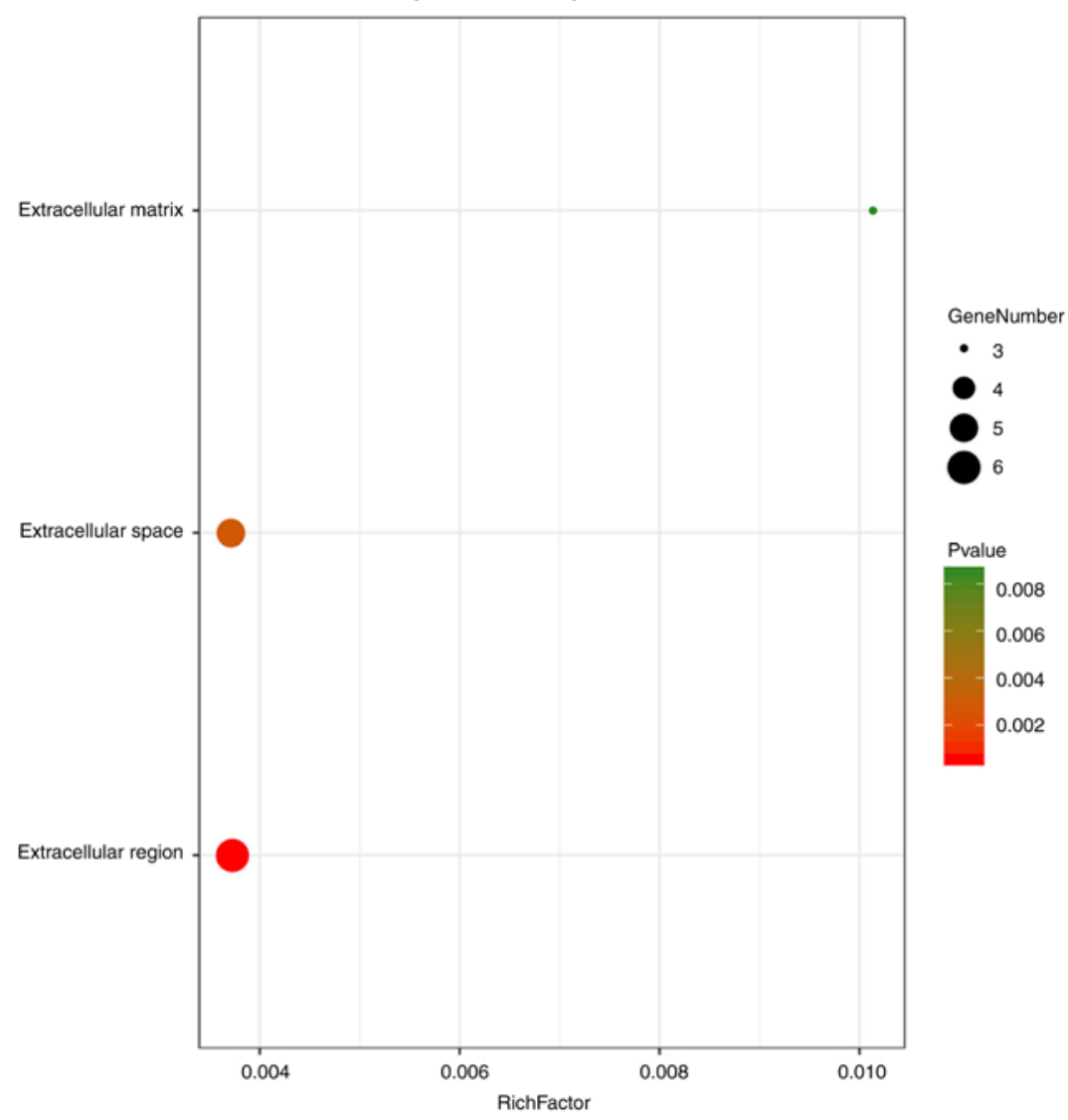

Figure 6. Enrichment analysis of the hub genes using the DAVID tool. Detailed information relating to changes in the (A) biological process, (B) cellular component among the hub genes. 
C

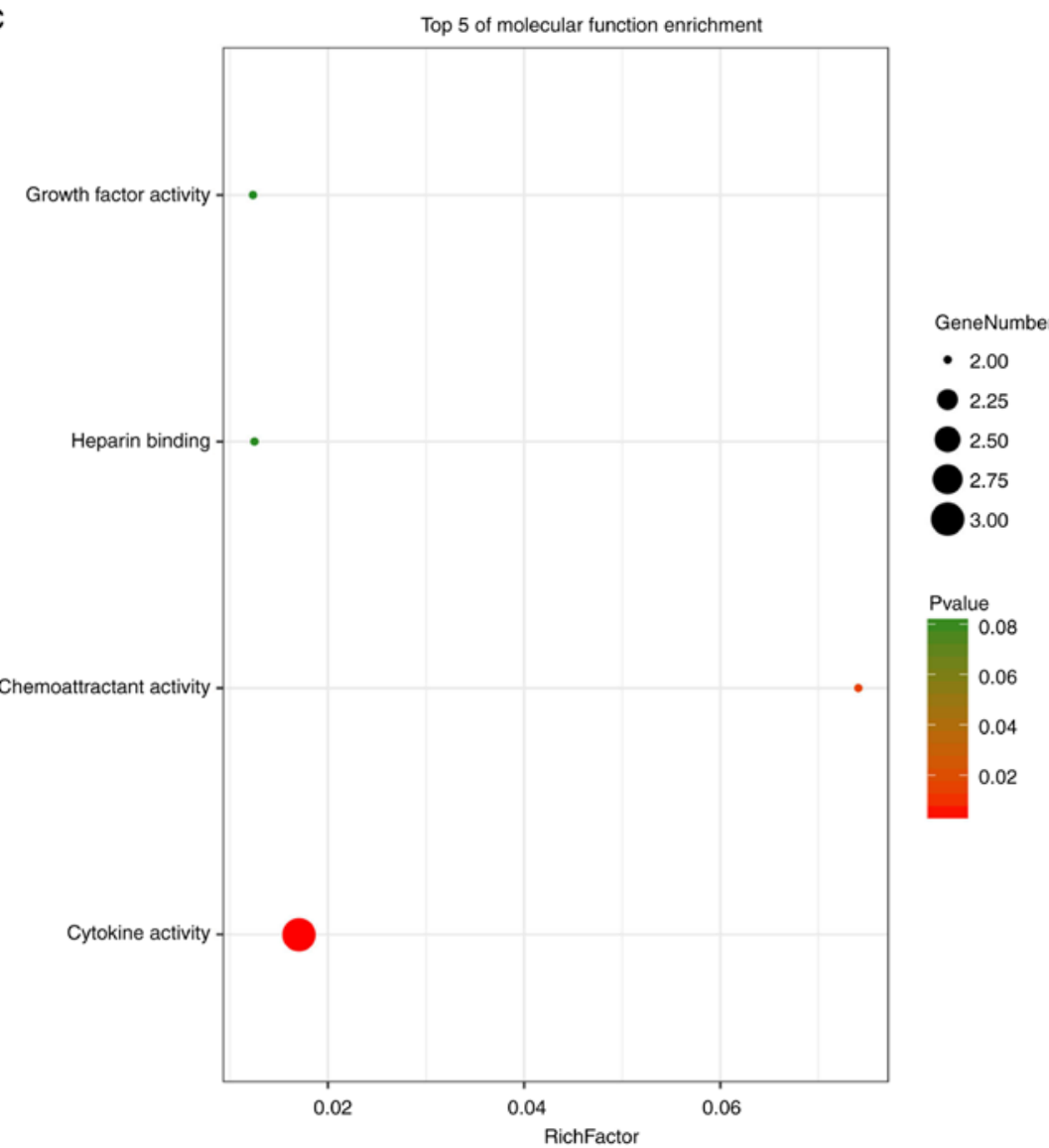

D

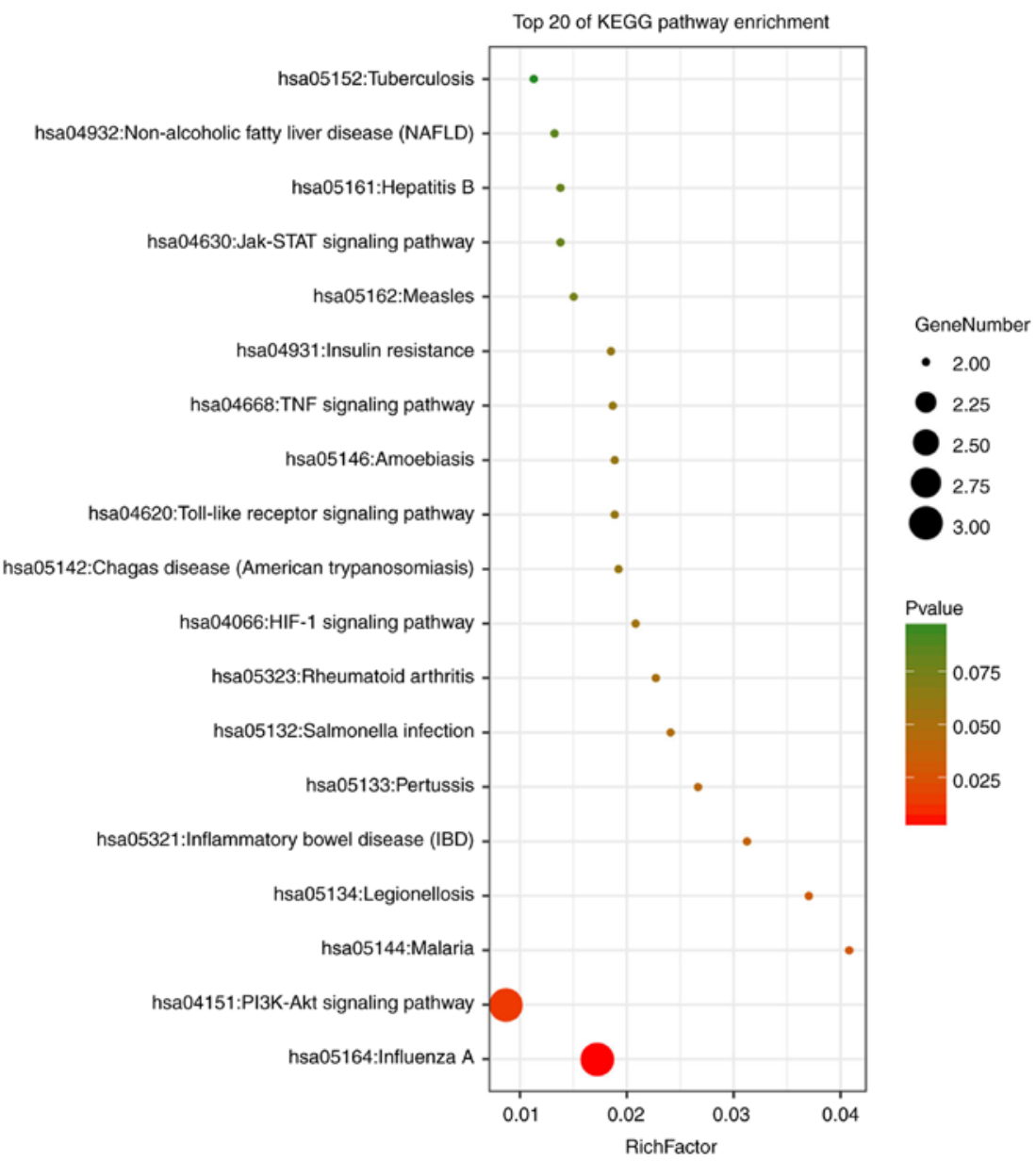

Figure 6. Continued. Enrichment analysis of the hub genes using the DAVID tool. Detailed information relating to changes in the (C) molecular function of differentially expressed genes among the hub genes. (D) KEGG pathway analysis for hub genes. KEGG, Kyoto Encyclopedia of Genes and Genomes. 
A

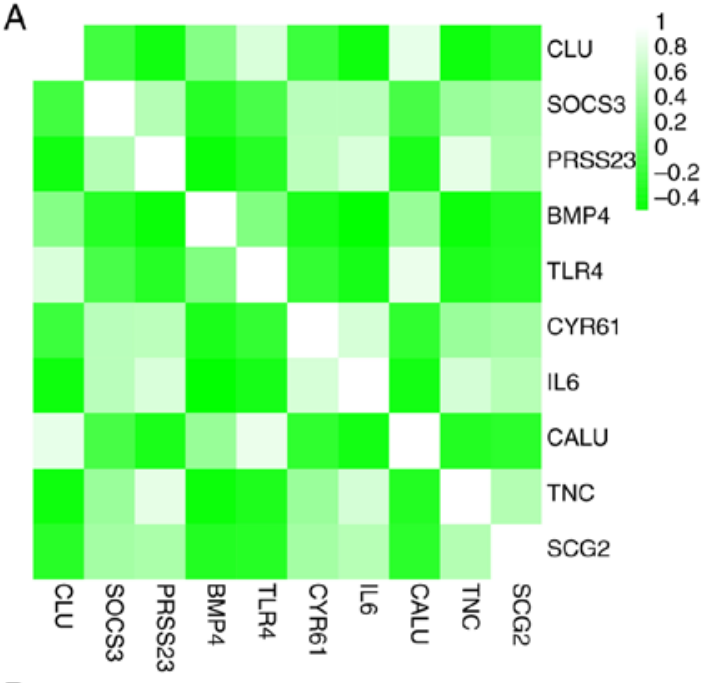

B

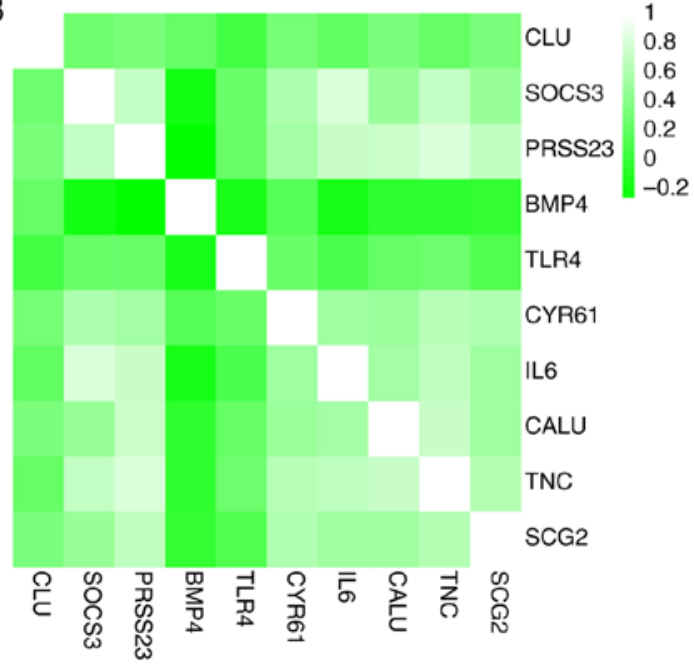

Figure 7. Correlation analysis among the hub genes. Heat maps showing the correlations between hub genes in the (A) GSE95065 and (B) GSE76885 datasets. The color reflects the intensity of the correlation. When $0<$ correlation $<1$, there exists a positive correlation. When $-1<$ correlation $<0$, there exists a negative correlation. The larger the absolute value of a number the stronger the correlation. IL6, interleukin-6; BMP4, bone morphogenetic protein 4; CALU, calumenin; CLU, clusterin; CYR61, cysteine rich angiogenic inducer 61; PRSS23, serine protease 23; SCG2, secretogranin II; SOCS3, suppressor of cytokine signaling 3; TLR4, Toll-like receptor 4; TNC, tenascin $\mathrm{C}$.

pivotal role in the amplification and cascading of the inflammatory response $(27,28)$. Bhattacharyya et al $(25,30)$ and Bhattacharyya and Varga (29) suggested that repeated injuries caused by chemical, infectious, mechanical, or autoimmune factors in genetically susceptible individuals produce DAMPs such as fibronectin-EDA and tenascin-C. These dangerous signals can be recognized by TLR4, dramatically augmenting the intensity of downstream signaling. On one hand, the increased expression of multiple genes involved in tissue remodeling in mesenchymal cells and the differentiation of myofibroblasts was elicited and fibroblasts were sensitized. On the other hand, the synergetic transforming growth factor also produced a fibrogenic effect. The elevated levels of tenascin-C in SSc skin biopsy samples are congruent with the 'DAMP hypothesis' (31). However, it is worth noting that the conclusion that numerous DAMPs are endogenous ligands of TLR4 was
Table II. Receiver operator characteristic curve analysis of hub gene expression for SSc.

\begin{tabular}{lcccr}
\hline & \multicolumn{4}{c}{ SSc } \\
\cline { 2 - 5 } Gene symbol & AUC & P-value & $95 \%$ CI & \multicolumn{1}{c}{ ODT } \\
\hline IL6 & 0.970 & $0.000^{\mathrm{c}}$ & $0.889-1.000$ & 40.502 \\
TLR4 & 0.822 & $0.002^{\mathrm{b}}$ & $0.733-1.000$ & 69.185 \\
CYR61 & 0.878 & $0.000^{\mathrm{c}}$ & $0.867-0.889$ & 584.379 \\
TNC & 0.852 & $0.001^{\mathrm{b}}$ & $0.722-0.933$ & 1128.397 \\
SCG2 & 0.856 & $0.001^{\mathrm{b}}$ & $0.800-0.833$ & 47.679 \\
SOCS3 & 0.759 & $0.011^{\mathrm{a}}$ & $0.667-0.833$ & 28.669 \\
BMP4 & 0.744 & $0.017^{\mathrm{a}}$ & $0.533-0.889$ & 112.929 \\
CALU & 0.744 & $0.017^{\mathrm{a}}$ & $0.733-1.000$ & 510.164 \\
PRSS23 & 0.996 & $0.000^{\mathrm{c}}$ & $0.944-1.000$ & 910.976 \\
CLU & 0.863 & $0.000^{\mathrm{c}}$ & $0.733-0.944$ & 1308.718 \\
\hline
\end{tabular}

${ }^{\mathrm{a}-\mathrm{c}}$ Significant variables. ${ }^{\mathrm{a}} \mathrm{P}<0.05,{ }^{\mathrm{b}} \mathrm{P}<0.01,{ }^{\mathrm{c}} \mathrm{P}<0.001$. AUC, area under curve; ${ }^{\text {max }}$ the maximum of AUC; ODT, optimal diagnostic threshold; SSc, systemic sclerosis; CI, confidence interval; IL6, interleukin-6; BMP4, bone morphogenetic protein 4; CALU, calumenin; CLU, clusterin; CYR61, cysteine rich angiogenic inducer 61; PRSS23, serine protease 23; SCG2, secretogranin II; SOCS3, suppressor of cytokine signaling 3; TLR4, Toll-like receptor 4; TNC, tenascin C.

based on in vitro immunoprecipitation and in vivo functional cell-based assays using TLR4 (-/-) mutant mice. Additionally there have been no reports relating to the complex crystal structure of DAMP-TLR4 to confirm any direct interaction between DAMP and TLR4 (30). Furthermore, there are 'contradictory' observations on the expression of TNC according to the results of the present study.

Stifano et al (32) performed skin biopsies of the dorsal forearm from 24 patients with dcSSc and $11 \mathrm{HC}$ patients and tested the samples using RNA isolation and quantitative PCR. The results revealed that the expression of TLR4 mRNA in patients with dcSSc was significantly increased compared with the control group. Bhattacharyya et al (25) performed immunohistochemistry on the forearm from 19 patients with $\mathrm{dcSSc}$ and 11 cases of HC. They drew a similar conclusion and semi-quantitative analysis of TLR4 expression confirmed it. The present study, however, combined two different microarray datasets for analysis and showed that TLR4 expression was low in patients with SSc. This indicates that the expression of TLR4 in patients with SSc is not straightforward. This may be due to sampling being performed at different stages of the disease process or sites of the skin, but the role of TLR4 in the pathogenesis of SSc may also be investigated from other perspectives. Yang et al (33) found that genetic or pharmacological inhibition of TLR4 promoted the formation of a local immunosuppressive microenvironment and attenuated autophagy-associated degradation of collagen and cell death in the fibrotic lung tissues, and thus led to pulmonary inflammation, fibrosis, and dysfunction induced by bleomycin being aggravated, which eventually caused the death of the experimental animals. In contrast, activation of TLR4 rapidly ended acute inflammation, reversed any pulmonary fibrosis 
A

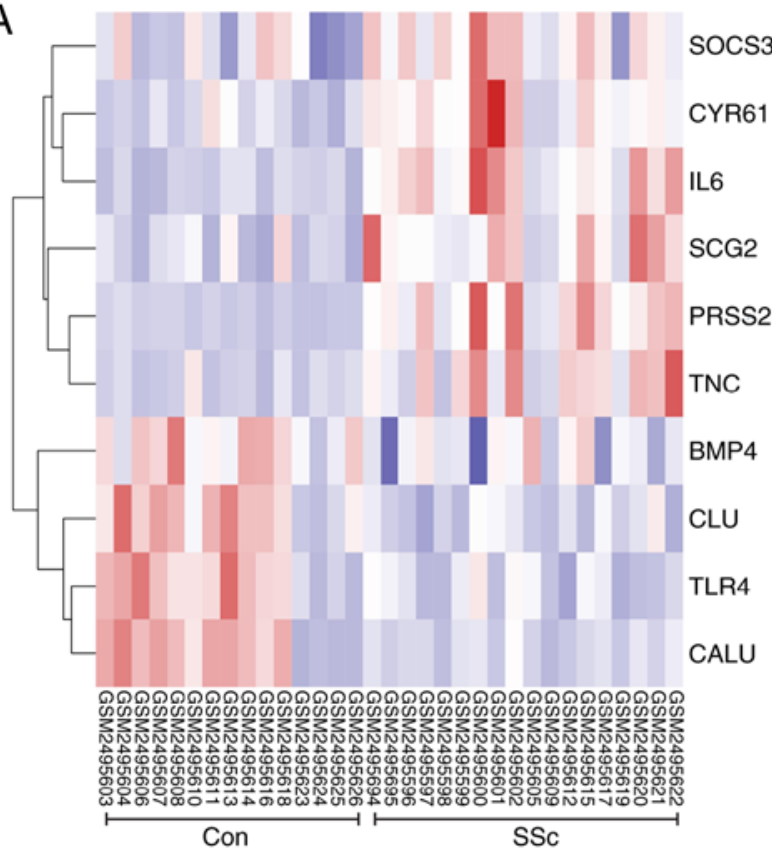

C

3
2
1
0
-1
-2
-3

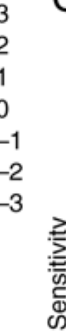

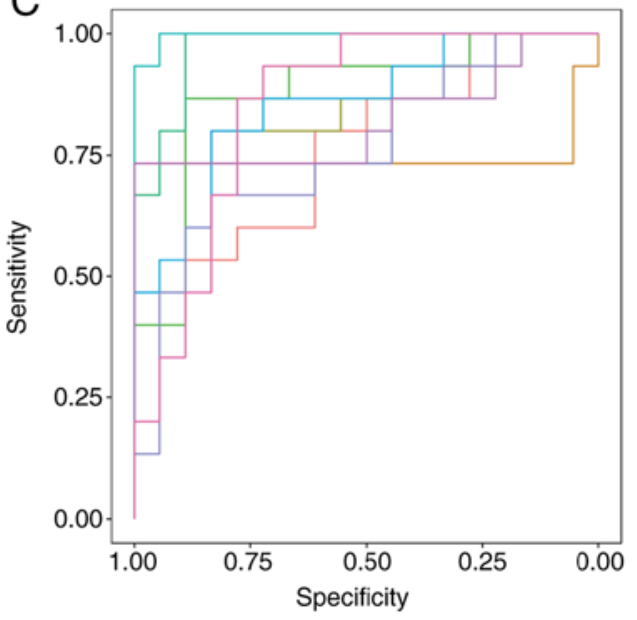

Name

- BMP4

- CALU

- CLU

- CYR6

- IL6

- PRSS23

- SCG2

- SOCS3

- TLR4

- TNC

B

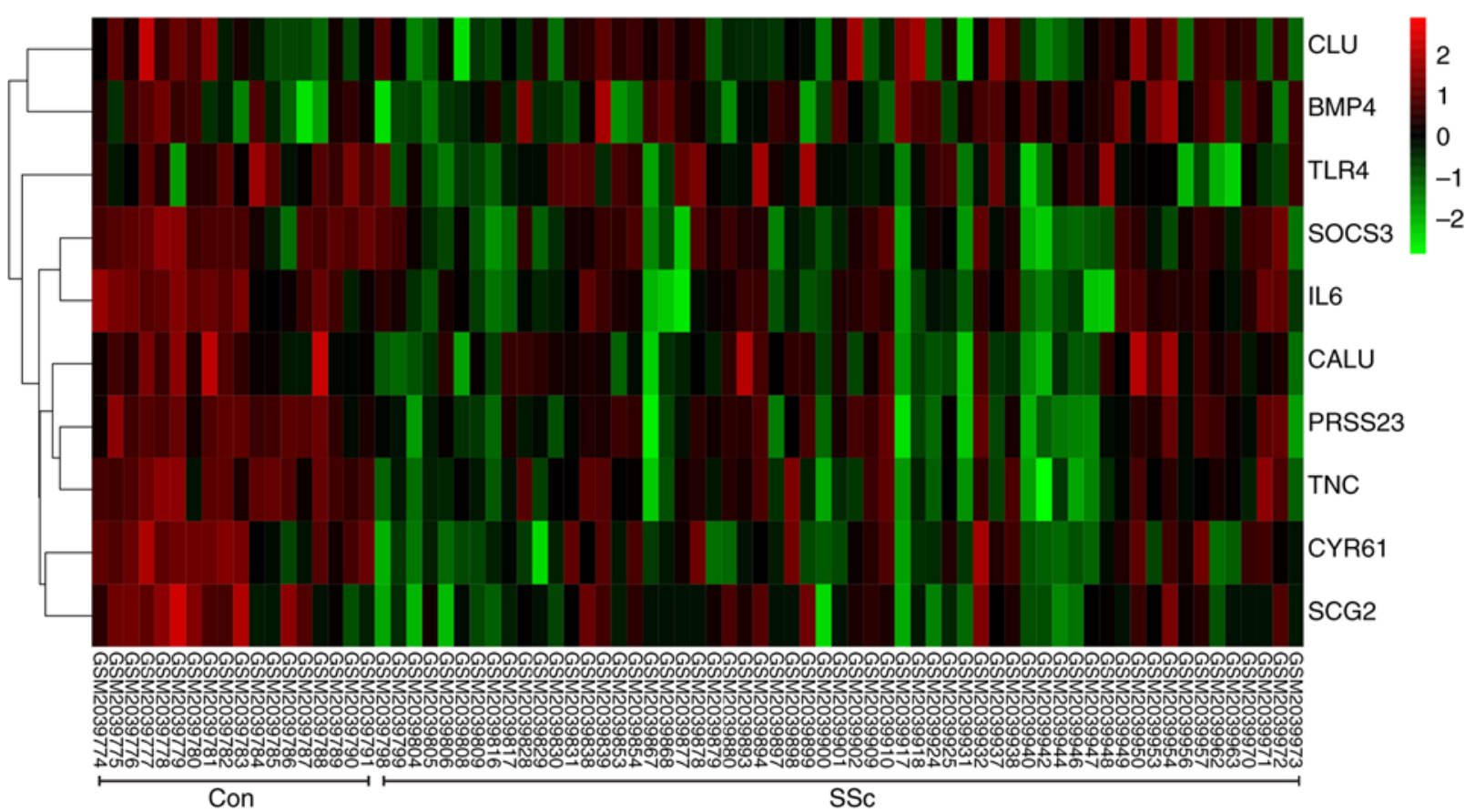

Figure 8. Expression analysis and ROC curves of all the hub genes. (A) A hierarchical clustering heat-map of the hub genes from control and SSc skin tissue from the GSE95065 dataset. The X-axis represents sample symbols (from left to right: Samples of the control skin tissue and samples of SSc skin tissue); the Y-axis represents differentially expressed genes. Blue, low expression; white, medium expression; and red, high expression. (B) A hierarchical clustering heat-map of the hub genes from control and SSc skin tissue from the GSE76885 dataset. The X-axis represents sample symbols (from left to right: Samples of the control skin tissue and samples of SSc skin tissue); the Y-axis represents differentially expressed genes. Green, low expression; black, medium expression; and red, high expression. (C) ROC curves indicating that all hub genes could sensitively and specifically predict SSc. ROC, receiver operator characteristic; SSc, systemic scelerosis. IL6, interleukin-6; BMP4, bone morphogenetic protein 4; CALU, calumenin; CLU, clusterin; CYR61, cysteine rich angiogenic inducer 61; PRSS23, serine protease 23; SCG2, secretogranin II; SOCS3, suppressor of cytokine signaling 3; TLR4, Toll-like receptor 4; TNC, tenascin C.

that occurred and improved lung function. Similarly, blocking TLR4 can impair the resolution of silica-induced chronic inflammation and fibrosis (33). Thus, as a crucial component of the innate immune system, the counter-regulation of TLR4 is likely to be a self-protection mechanism initiated during defense reactions and both its over-activation and deficiency may exacerbate inflammation and fibrosis. It might be of great significance to investigate which condition is predominant in SSc at its different stages and in SSc with pulmonary or other organ fibrosis.

IL-6 is a pleiotropic cytokine that plays a crucial role in immune regulation and inflammation. Excessive IL-6 increases the secretion of immunoglobulin and the production of autoantibodies. In murine models, IL- 6 plays an key role in 
A

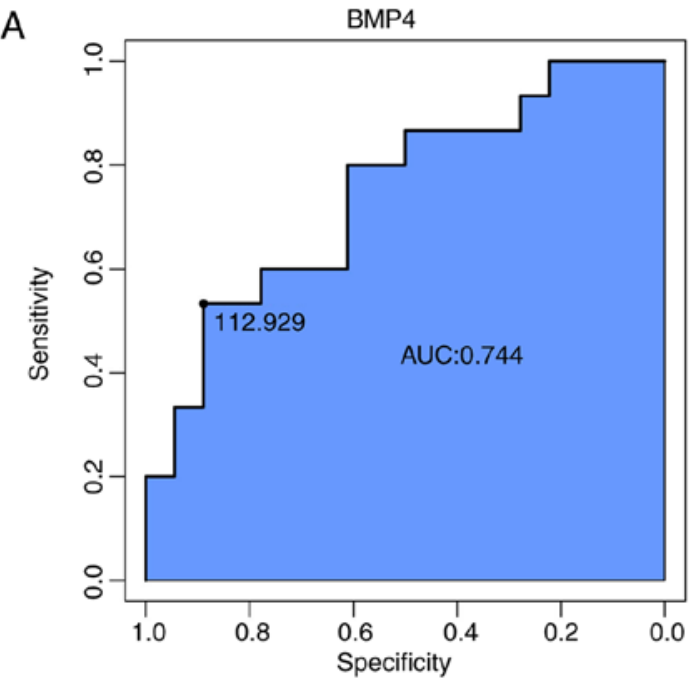

C

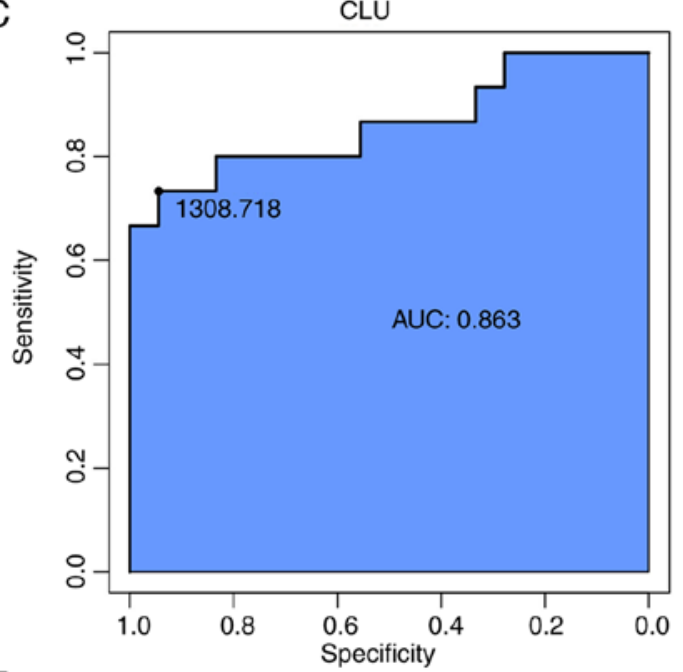

$\mathrm{E}$

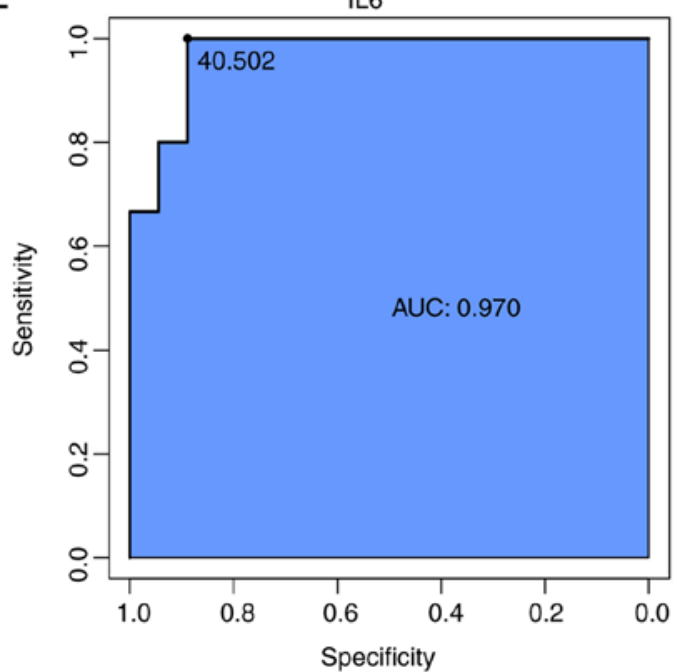

B

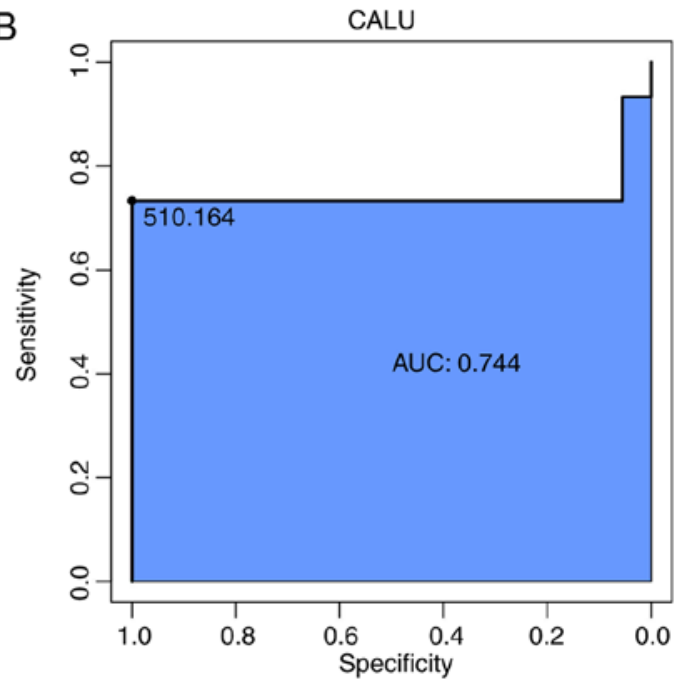

D

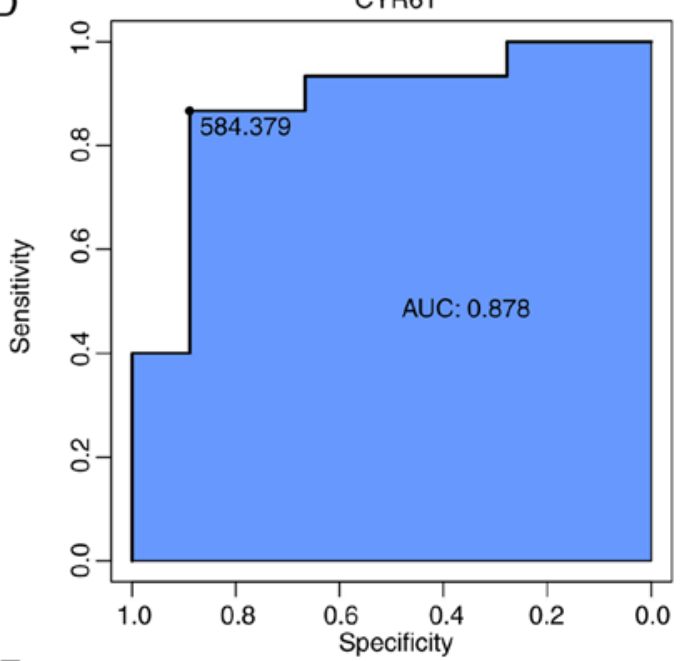

$\mathrm{F}$

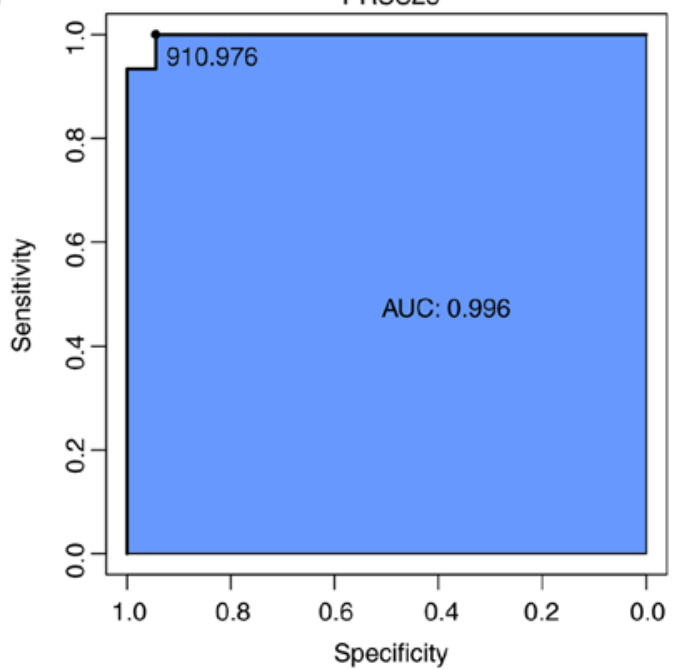

Figure 9. The respective receiver operator characteristic curves of per hub genes. (A) BMP4, (B) CALU, (C) CLU, (D) CYR61, (E) IL6, (F) PRSS23. AUC, area under the curve; BMP4, bone morphogenetic protein 4; CALU, calumenin; CLU, clusterin; CYR61, cysteine rich angiogenic inducer 61; IL6, interleukin-6; PRSS23, serine protease 23 .

the differentiation of cytotoxic $\mathrm{T}$ cells and $\mathrm{T}$ helper 17 cells in addition to its B-cell stimulatory effect $(34,35)$. Although its role in SSc has not yet been determined, increased IL-6 expression in the skin has been observed in SSc, as was shown in the present study (36). Furthermore, anti-IL-6 antibody treatment suppressed procollagen production in SSc-affected fibroblasts in vitro (37). In the bleomycin-induced SSc models, genetic deletion of IL-6 reduced myofibroblasts numbers and resulted in remission of the disease (38). Elevated serum IL-6 levels were also correlated with modified Rodnan total skin 
G

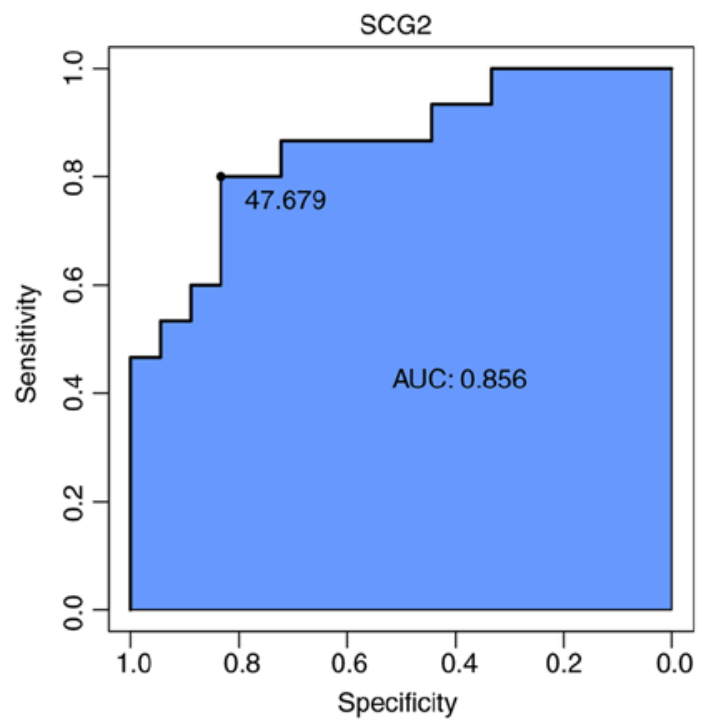

I

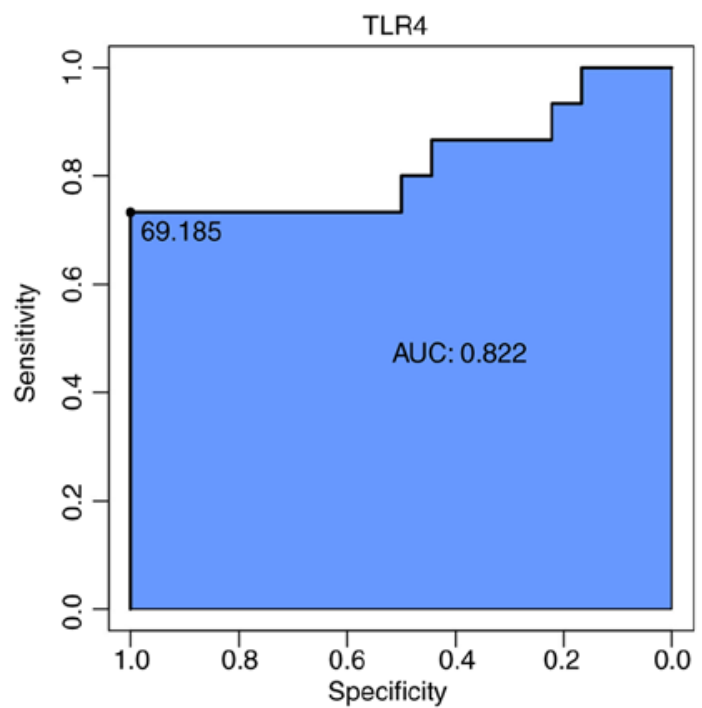

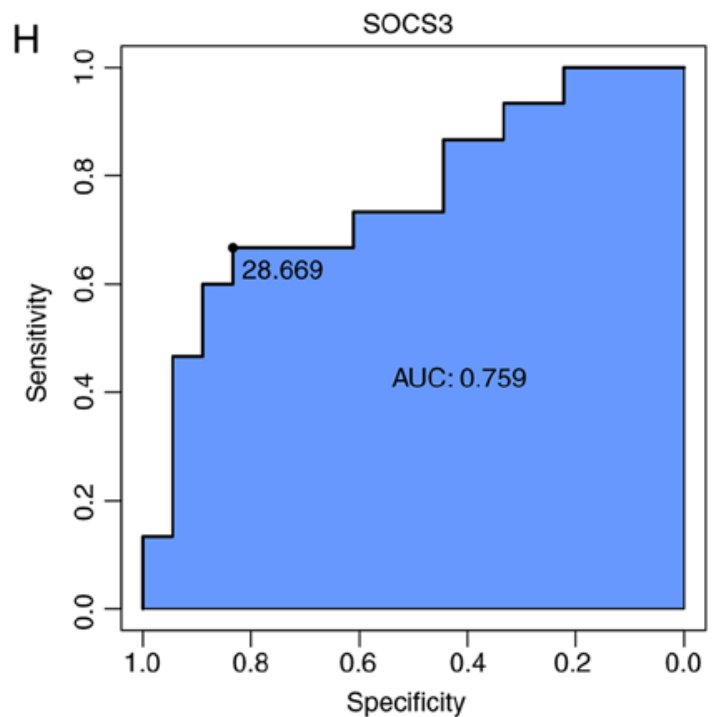

J

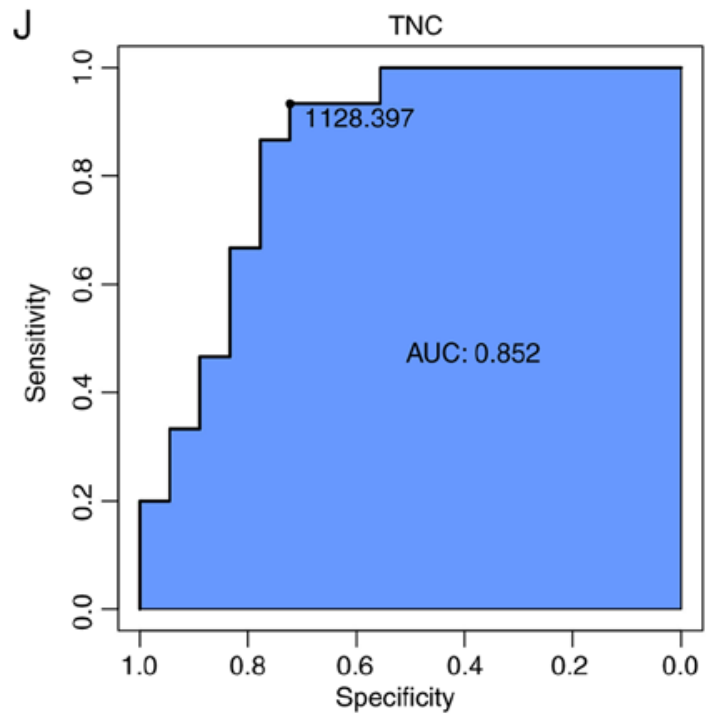

Figure 9. Continued. The respective receiver operator characteristic curves of per hub genes. (G) SCG2, (H) SOCS3, (I) TLR4, (J) TNC. AUC, area under the curve; SCG2, secretogranin II; SOCS3, suppressor of cytokine signaling 3; TLR4, Toll-like receptor 4; TNC, tenascin C.

scores in SSc patients and elevated IL-6 in bronchoalveolar lavage fluid of SSc patients is thought to relate to pulmonary fibrosis $(39,40)$. Most importantly, the faSScinate study, a phase II trial, demonstrated a significant improvement of skin sclerosis in progressive dcSSc patients with IL-6-related inflammatory signs after 24 weeks of administration of tocilizumab (41). Recent findings indicate that tocilizumab has the potential to modify SSc vasculopathy. To further validate tocilizumab efficacy, a global phase III trial is currently under way (42).

The present study also found that CALU expression was low in patients with SSc. The coding product of CALU is calumenin, which belongs to the CREC protein family, the EF-hand calcium-binding proteins localizes to multiple sites of the secretory pathway of mammalian cells (e.g., endoplasmic reticulum, Golgi apparatus and the extracellular matrix). Of these proteins, calumenin is the only member reported as likely to be exocytic $(43,44)$. Additionally calumenin was revealed to be associated with malignant cell transformation and metastasis (45-47). Vorum et al (48) suggested that calumenin might play a role in the immune defense system because it interacted with the $\mathrm{P}$ component of serum amyloid protein. Further research is needed into the function of CALU in immune responses and its role in the set of SSc.

Although the work described here involved rigorous bioinformatics analysis, it has some limitations. First, the sample size was relatively small and analysis of a larger data sample is necessary to verify the results. Second, the results of this study are based on an in silico analysis and molecular and experimental validation is necessary.

In conclusion, using bioinformatics technologies two datasets from the GEO database were integrated for analysis and obtained 106 DEGs and 10 hub genes related to SSc (CLU, SOCS3, PRSS23, BMP4, TLR4, CYR61, IL6, CALU, TNC, and SCG2), with TLR4, IL6 and CALU being particularly prominent. There are several contradictions and ambiguities relating to the roles played by TLR4 in SSc, which are waiting for further explanation. Notably there have been no previous reports of CALU being associated with SSc. Therefore, the present study suggests that more studies should be conducted 
to explore and elucidate these areas, so as to provide new ideas and targets for the diagnosis and treatment of SSc.

\section{Acknowledgements}

The authors gratefully thank the Department of Rheumatology in Beijing Hospital for technical assistance.

\section{Funding}

This study was funded by the National Natural Science Foundation of China (grant. no. 31140008) and the Beijng Hospital Research Foundation (grant. no. BJ-2014-033).

\section{Availability of data and materials}

The datasets used and/or analyzed during the current study are available from the corresponding author on reasonable request.

\section{Authors' contributions}

CX and LM conceived and designed the study. CX was a major contributor in writing the manuscript and submitting the manuscript. YD collected the datasets; YC analyzed the data; $\mathrm{CZ}$ and $\mathrm{CH}$ made substantial contributions to research conception; $\mathrm{XZ}$ and $\mathrm{CH}$ designed the draft of the research process. $\mathrm{CH}$ had been involved in revising manuscript critically for important intellectual content. All authors read and approved the final manuscript.

\section{Ethics approval and consent to participate}

The data of this research was downloaded from the GEO database, a public website. All institutional and national guidelines for the care and use of participates were followed.

\section{Patient consent for publication}

Not applicable.

\section{Competing interests}

The authors declare that they have no competing interests.

\section{References}

1. Denton CP and Khanna D: Systemic sclerosis. Lancet 390: $1685-1699,2017$

2. Chifflot H, Fautrel B, Sordet C, Chatelus E and Sibilia J: Incidence and prevalence of systemic sclerosis: A systematic literature review. Semin Arthritis Rheum 37: 223-235, 2008.

3. Nihtyanova SI, Tang EC, Coghlan JG, Wells AU, Black CM and Denton CP: Improved survival in systemic sclerosis is associated with better ascertainment of internal organ disease: A retrospective cohort study. QJM 103: 109-115, 2010.

4. Tyndall AJ, Bannert B, Vonk M, Airo P, Cozzi F, Carreira PE, Bancel DF, Allanore Y, Muller-Ladner U, Distler O, et al: Causes and risk factors for death in systemic sclerosis: A study from the EULAR Scleroderma Trials and Research (EUSTAR) database. Ann Rheum Dis 69: 1809-1815, 2010.

5. Bossini-Castillo L, Lopez-Isac E, Mayes MD and Martin J: Genetics of systemic sclerosis. Semin Immunopathol 37: 443-451, 2015.

6. Salazar G and Mayes MD: Genetics, epigenetics, and genomics of systemic sclerosis. Rheum Dis Clin North Am 41: 345-366, 2015 .
7. Derrett-Smith EC, Martyanov V, Chighizola CB, Moinzadeh $P$ Campochiaro C, Khan K, Wood TA, Meroni PL, Abraham DJ, Ong VH, et al: Limited cutaneous systemic sclerosis skin demonstrates distinct molecular subsets separated by a cardiovascular development gene expression signature. Arthritis Res Ther 19: $156,2017$.

8. Gardner H, Shearstone JR, Bandaru R, Crowell T, Lynes M, Trojanowska M, Pannu J, Smith E, Jablonska S, Blaszczyk M, et al: Gene profiling of scleroderma skin reveals robust signatures of disease that are imperfectly reflected in the transcript profiles of explanted fibroblasts. Arthritis Rheum 54: 1961-1973, 2006.

9. Chouri E, Servaas NH, Bekker CPJ, Affandi AJ, Cossu M, Hillen MR, Angiolilli C, Mertens JS, van den Hoogen LL, Silva-Cardoso S, et al: Serum microRNA screening and functional studies reveal miR-483-5p as a potential driver of fibrosis in systemic sclerosis. J Autoimmun 89: 162-170, 2018.

10. Falzone L, Scola L, Zanghi A, Biondi A, Di Cataldo A, Libra $\mathrm{M}$ and Candido S: Integrated analysis of colorectal cancer microRNA datasets: Identification of microRNAs associated with tumor development. Aging (Albany NY) 10: 1000-1014, 2018.

11. Falzone L, Candido S, Salemi R, Basile MS, Scalisi A, McCubrey JA, Torino F, Signorelli SS, Montella M and Libra M: Computational identification of microRNAs associated to both epithelial to mesenchymal transition and NGAL/MMP-9 pathways in bladder cancer. Oncotarget 7: 72758-72766, 2016.

12. Hafsi S, Candido S, Maestro R, Falzone L, Soua Z, Bonavida B, Spandidos DA and Libra M: Correlation between the overexpression of Yin Yang 1 and the expression levels of miRNAs in Burkitt's lymphoma: A computational study. Oncol Lett 11: 1021-1025, 2016.

13. Falzone L, Lupo G, La Rosa GRM, Crimi S, Anfuso CD, Salemi R, Rapisarda E, Libra M and Candido S: Identification of novel MicroRNAs and their diagnostic and prognostic significance in oral cancer. Cancers (Basel) 11: E610, 2019.

14. Barrett T, Wilhite SE, Ledoux P, Evangelista C, Kim IF, Tomashevsky M, Marshall KA, Phillippy KH, Sherman PM, Holko M, et al: NCBI GEO: Archive for functional genomics data sets-update. Nucleic Acids Res 41 (Database Issue): D991-D995, 2013.

15. Edgar R, Domrachev $M$ and Lash AE: Gene Expression Omnibus: NCBI gene expression and hybridization array data repository. Nucleic Acids Res 30: 207-210, 2002.

16. Sun $\mathrm{YH}, \mathrm{Xie} \mathrm{M}, \mathrm{Wu} \mathrm{SD}, \mathrm{Zhang} \mathrm{J}$ and Huang CZ: Identification and interaction analysis of key genes and MicroRNAs in systemic sclerosis by bioinformatics approaches. Curr Med Sci 39: 645-652, 2019.

17. Ritchie ME, Phipson B, Wu D, Hu Y, Law CW, Shi W and Smyth GK: Limma powers differential expression analyses for RNA-sequencing and microarray studies. Nucleic Acids Res 43: e47, 2015 .

18. Huang DW, Sherman BT, Tan Q, Collins JR, Alvord WG, Roayaei J, Stephens R, Baseler MW, Lane HC and Lempicki RA: The DAVID gene functional classification tool: A novel biological module-centric algorithm to functionally analyze large gene lists. Genome Biol 8: R183, 2007.

19. Ashburner M, Ball CA, Blake JA, Botstein D, Butler $\mathrm{H}$ Cherry JM, Davis AP, Dolinski K, Dwight SS, Eppig JT, et al: Gene ontology: Tool for the unification of biology. The Gene Ontology Consortium. Nat Genet 25: 25-29, 2000.

20. Smoot ME, Ono K, Ruscheinski J, Wang PL and Ideker T: Cytoscape 2.8: New features for data integration and network visualization. Bioinformatics 27: 431-432, 2011.

21. Bader GD and Hogue CW: An automated method for finding molecular complexes in large protein interaction networks. BMC Bioinformatics 4: 2, 2003.

22. Young A and Khanna D: Systemic sclerosis: A systematic review on therapeutic management from 2011 to 2014. Curr Opin Rheumatol 27: 241-248, 2015.

23. Elhai M, Avouac J, Kahan A and Allanore Y: Systemic sclerosis: Recent insights. Joint Bone Spine 82: 148-153, 2015.

24. Takahashi T, Asano Y, Ichimura Y, Toyama T, Taniguchi T, Noda S, Akamata K, Tada Y, Sugaya M, Kadono T and Sato S: Amelioration of tissue fibrosis by toll-like receptor 4 knockout in murine models of systemic sclerosis. Arthritis Rheumatol 67: 254-265, 2015.

25. Bhattacharyya S, Kelley K, Melichian DS, Tamaki Z, Fang F, Su Y, Feng G, Pope RM, Budinger GR, Mutlu GM, et al: Toll-like receptor 4 signaling augments transforming growth factor- $\beta$ responses: A novel mechanism for maintaining and amplifying fibrosis in scleroderma. Am J Pathol 182: 192-205, 2013. 
26. Fineschi S, Goffin L, Rezzonico R, Cozzi F, Dayer JM, Meroni PL and Chizzolini C: Antifibroblast antibodies in systemic sclerosis induce fibroblasts to produce profibrotic chemokines, with partial exploitation of toll-like receptor 4. Arthritis Rheum 58: 3913-3923, 2008.

27. Molteni M, Gemma S and Rossetti C: The role of Toll-like receptor 4 in infectious and noninfectious inflammation. Mediators Inflamm 2016: 6978936, 2016.

28. Barton GM and Medzhitov R: Toll-like receptor signaling pathways. Science 300: 1524-1525, 2003.

29. Bhattacharyya S and Varga J: Endogenous ligands of TLR4 promote unresolving tissue fibrosis: Implications for systemic sclerosis and its targeted therapy. Immunol Lett 195: 9-17, 2018.

30. Bhattacharyya S, Midwood KS, Yin H and Varga J: Toll-like receptor-4 signaling drives persistent fibroblast activation and prevents fibrosis resolution in scleroderma. Adv Wound Care (New Rochelle) 6: 356-369, 2017.

31. Bhattacharyya S, Wang W, Morales-Nebreda L, Feng G, Wu M, Zhou X,Lafyatis R,Lee J,Hinchcliff M,Feghali-Bostwick C, et al: Tenascin-C drives persistence of organ fibrosis. Nat Commun 7: 11703, 2016.

32. Stifano G, Affandi AJ, Mathes AL, Rice LM, Nakerakanti S, Nazari B, Lee J, Christmann RB and Lafyatis R: Chronic Toll-like receptor 4 stimulation in skin induces inflammation, macrophage activation, transforming growth factor beta signature gene expression, and fibrosis. Arthritis Res Ther 16: R136, 2014.

33. Yang HZ, Wang JP, Mi S, Liu HZ, Cui B, Yan HM, Yan J, Li Z, Liu H, Hua F, et al: TLR4 activity is required in the resolution of pulmonary inflammation and fibrosis after acute and chronic lung injury. Am J Pathol 180: 275-292, 2012.

34. Bettelli E, Carrier Y, Gao W, Korn T, Strom TB, Oukka M, Weiner HL and Kuchroo VK: Reciprocal developmental pathways for the generation of pathogenic effector $\mathrm{TH} 17$ and regulatory T cells. Nature 441: 235-238, 2006.

35. Mangan PR, Harrington LE, O'Quinn DB, Helms WS, Bullard DC, Elson CO, Hatton RD, Wahl SM, Schoeb TR and Weaver CT: Transforming growth factor-beta induces development of the $\mathrm{T}(\mathrm{H}) 17$ lineage. Nature 441: 231-234, 2006.

36. Koch AE, Kronfeld-Harrington LB, Szekanecz Z, Cho MM, Haines GK, Harlow LA, Strieter RM, Kunkel SL, Massa MC, Barr WG, et al: In situ expression of cytokines and cellular adhesion molecules in the skin of patients with systemic sclerosis. Their role in early and late disease. Pathobiology 61: 239-246, 1993.

37. Kawaguchi Y, Hara M and Wright TM: Endogenous IL-1alpha from systemic sclerosis fibroblasts induces IL-6 and PDGF-A. J Clin Invest 103: 1253-1260, 1999.

38. Kitaba S, Murota H, Terao M, Azukizawa H, Terabe F, Shima Y, Fujimoto M, Tanaka T, Naka T, Kishimoto T and Katayama I: Blockade of interleukin- 6 receptor alleviates disease in mouse model of scleroderma. Am J Pathol 180: 165-176, 2012.
39. Sato S, Hasegawa M and Takehara K: Serum levels of interleukin- 6 and interleukin-10 correlate with total skin thickness score in patients with systemic sclerosis. J Dermatol Sci 27: 140-146, 2001.

40. Gudbjörnsson B, Hällgren R, Nettelbladt O, Gustafsson R, Mattsson A, af Geijerstam E and Totterman TH: Phenotypic and functional activation of alveolar macrophages, T lymphocytes and NK cells in patients with systemic sclerosis and primary Sjogren's syndrome. Ann Rheum Dis 53: 574-579, 1994.

41. Khanna D, Denton CP, Jahreis A, van Laar JM, Frech TM, Anderson ME, Baron M, Chung L, Fierlbeck G, Lakshminarayanan S, et al: Safety and efficacy of subcutaneous tocilizumab in adults with systemic sclerosis (faSScinate): A phase 2, randomised, controlled trial. Lancet 387: 2630-2640, 2016.

42. Taniguchi T, Asano Y, Fukasawa T, Yoshizaki A and Sato S: Critical contribution of the interleukin-6/signal transducer and activator of transcription 3 axis to vasculopathy associated with systemic sclerosis. J Dermatol 44: 967-971, 2017.

43. Honore B and Vorum H: The CREC family, a novel family of multiple EF-hand, low-affinity $\mathrm{Ca}(2+)$-binding proteins localised to the secretory pathway of mammalian cells. FEBS Lett 466 : 11-18, 2000.

44. Vorum H, Liu X, Madsen P, Rasmussen HH and Honore B: Molecular cloning of a cDNA encoding human calumenin, expression in Escherichia coli and analysis of its $\mathrm{Ca} 2+$-binding activity. Biochim Biophys Acta 1386: 121-131, 1998.

45. Nimmrich I, Erdmann S, Melchers U, Finke U, Hentsch S, Moyer MP, Hoffmann I and Muller O: Seven genes that are differentially transcribed in colorectal tumor cell lines. Cancer Lett 160: 37-43, 2000.

46. Nagano K, Imai S, Zhao X, Yamashita T, Yoshioka Y, Abe Y, Mukai Y, Kamada H, Nakagawa S, Tsutsumi Y and Tsunoda S: Identification and evaluation of metastasis-related proteins, oxysterol binding protein-like 5 and calumenin, in lung tumors. Int J Oncol 47: 195-203, 2015.

47. Wang Q, Shen B, Chen L, Zheng P, Feng H, Hao Q, Liu X, Liu L, $\mathrm{Xu}$ S, Chen J and Teng J: Extracellular calumenin suppresses ERK1/2 signaling and cell migration by protecting fibulin- 1 from MMP-13-mediated proteolysis. Oncogene 34: 1006-1018, 2015.

48. Vorum H, Jacobsen C and Honore B: Calumenin interacts with serum amyloid P component. FEBS Lett 465: 129-134, 2000.

49. Miyakoshi K, Murphy MJ, Yeoman RR, Mitra S, Dubay CJ and Hennebold JD: The identification of novel ovarian proteases through the use of genomic and bioinformatic methodologies. Biol Reprod 75: 823-835, 2006.

This work is licensed under a Creative Commons Attribution-NonCommercial-NoDerivatives 4.0 International (CC BY-NC-ND 4.0) License. 